\title{
DISTINCT CHEMICAL REGIONS IN THE “PRESTELLAR” INFRARED DARK CLOUD G028.23-00.19
}

\author{
Patricio Sanhueza ${ }^{1}$, James M. Jackson ${ }^{1}$, Jonathan B. Foster ${ }^{2}$, Izaskun Jimenez-Serra ${ }^{3}$, \\ William J. Dirienzo ${ }^{4}$, AND Thushara Pillai ${ }^{5}$ \\ ${ }^{1}$ Institute for Astrophysical Research, Boston University, Boston, MA 02215, USA; patricio@bu.edu \\ ${ }^{2}$ Yale Center for Astronomy and Astrophysics, Yale University, New Haven, CT 06520, USA \\ ${ }^{3}$ European Southern Observatory, Karl-Schwarzschild-Str. 2, D-85748 Garching, Germany \\ ${ }^{4}$ Department of Astronomy, University of Virginia, P.O. Box 3818, Charlottesville, VA 22903, USA \\ ${ }^{5}$ Caltech, MC 249-17, 1200 East California Boulevard, Pasadena, CA 91125, USA \\ Received 2013 February 13; accepted 2013 June 29; published 2013 August 1
}

\begin{abstract}
We have observed the Infrared Dark Cloud (IRDC) G028.23-00.19 at $3.3 \mathrm{~mm}$ using the Combined Array for Research in Millimeter-wave Astronomy. In its center, the IRDC hosts one of the most massive $\left(\sim 1520 M_{\odot}\right)$ quiescent, cold $\left(12 \mathrm{~K}\right.$ ) clumps known (MM1). The low temperature, high $\mathrm{NH}_{2} \mathrm{D}$ abundance, narrow molecular line widths, and absence of embedded infrared sources (from 3.6 to $70 \mu \mathrm{m}$ ) indicate that the clump is likely prestellar. Strong $\mathrm{SiO}$ emission with broad line widths $\left(6-9 \mathrm{~km} \mathrm{~s}^{-1}\right)$ and high abundances $\left((0.8-4) \times 10^{-9}\right)$ is detected in the northern and southern regions of the IRDC, unassociated with MM1. We suggest that $\mathrm{SiO}$ is released to the gas phase from the dust grains through shocks produced by outflows from undetected intermediate-mass stars or clusters of low-mass stars deeply embedded in the IRDC. A weaker $\mathrm{SiO}$ component with narrow line widths $\left(\sim 2 \mathrm{~km} \mathrm{~s}^{-1}\right)$ and low abundances $\left(4.3 \times 10^{-11}\right)$ is detected in the center-west region, consistent with either a "subcloud-subcloud" collision or an unresolved population of a few low-mass stars. We report widespread $\mathrm{CH}_{3} \mathrm{OH}$ emission throughout the whole IRDC and the first detection of extended narrow methanol emission $\left(\sim 2 \mathrm{~km} \mathrm{~s}^{-1}\right)$ in a cold, massive prestellar clump (MM1). We suggest that the most likely mechanism releasing methanol into the gas phase in such a cold region is the exothermicity of grain-surface reactions. $\mathrm{HN}^{13} \mathrm{C}$ reveals that the IRDC is actually composed of two distinct substructures ("subclouds") separated in velocity space by $\sim 1.4 \mathrm{~km} \mathrm{~s}^{-1}$. The narrow SiO component arises where the subclouds overlap. The spatial distribution of $\mathrm{C}_{2} \mathrm{H}$ resembles that of $\mathrm{NH}_{2} \mathrm{D}$, which suggests that $\mathrm{C}_{2} \mathrm{H}$ also traces cold gas in this IRDC.
\end{abstract}

Key words: astrochemistry - ISM: abundances - ISM: clouds - ISM: molecules - stars: formation

\section{INTRODUCTION}

High-mass stars play a key role in the evolution of the energetics and chemistry of molecular clouds and galaxies. However, our knowledge of their formation is still poor, compared with that of low-mass stars. There are several factors that make the study of high-mass star formation more challenging than that of their low-mass counterparts. High-mass stars are rare and evolve quickly. Massive star-forming regions in the earliest stages of evolution are mostly located at large distances $(\gtrsim 3 \mathrm{kpc})$ and are heavily extincted by dust. The earliest well-understood phase of high-mass star formation is the hot molecular cores (e.g., Garay \& Lizano 1999). However, at this stage, the high-mass stars have already begun burning hydrogen and reached the zero-age main sequence, disturbing and changing the kinematics and chemistry of the initially starless cocoon.

Prestellar cores represent the earliest stage of star formation. These starless cores are formed by dense, centrally concentrated parcels of gravitationally bound gas (e.g., André et al. 2009). Prestellar cores will eventually collapse and evolve to form stars. The prestellar phase in high-mass star formation has been very difficult to find. Initially, large infrared (IR)/millimeter surveys had poor angular resolution and sensitivity, producing a bias toward bright, evolved objects and a misidentification of starless candidates. However, with the advent of new space telescopes, such as Spitzer and Herschel, and submillimeter Galactic plane surveys, it has been possible to study in detail the best candidates for dense regions which will eventually form massive stars, the so-called Infrared Dark Clouds (IRDCs). IRDCs appear as dark silhouettes against the Galactic mid-IR background. Galactic plane surveys revealed thousands of IRDCs (Infrared Space Observatory, Perault et al. 1996; Midcourse Space Experiment, Egan et al. 1998; Simon et al. 2006; Spitzer, Peretto \& Fuller 2009; Kim et al. 2010). The first studies characterizing them suggested that they were cold $(<25 \mathrm{~K})$, massive $\left(\sim 10^{2}-10^{4} M_{\odot}\right)$, and dense $\left(\gtrsim 10^{5} \mathrm{~cm}^{-3}\right)$ molecular clouds with high column densities $\left(\sim 10^{23} \mathrm{~cm}^{-2}\right.$; Carey et al. 1998, 2000). More recent studies on IRDC clumps ${ }^{6}$ show that they have typical masses of $\sim 120 M_{\odot}$, sizes of $\sim 0.5 \mathrm{pc}$ (Rathborne et al. 2006), dust temperatures that range between 16 and $52 \mathrm{~K}$, and luminosities that range from $\sim 10$ to $5 \times 10^{4} L_{\odot}$ (Rathborne et al. 2010). $\mathrm{NH}_{3}$ rotational temperatures are typically lower than dust temperatures and range from $\sim 10$ to $20 \mathrm{~K}$ (Pillai et al. 2006; Sakai et al. 2008; Devine et al. 2011; Ragan et al. 2011).

Evidence of active high-mass star formation in IRDCs is inferred by the presence of ultracompact $\mathrm{H}$ II regions (Battersby et al. 2010), hot cores (Rathborne et al. 2008), embedded $24 \mu \mathrm{m}$ sources (Chambers et al. 2009), molecular outflows (Sanhueza et al. 2010; Wang et al. 2011), or maser emission (Wang et al. 2006; Chambers et al. 2009). IRDCs also host massive, cold IR-dark clumps that show similar physical properties to those clumps that host active high-mass star formation, except that the temperature and luminosity are lower. These characteristics suggest that massive, cold IR-dark clumps will form high-mass stars in the future. These objects are the best candidates for the

\footnotetext{
6 Throughout this paper, we use the term "clump" to refer to a dense object within an IRDC with a size of the order $\sim 0.1-1 \mathrm{pc}$ and a mass $\sim 10^{2}-10^{3} M_{\odot}$. We use the term "core" to describe a compact, dense object within a clump with a size $\sim 0.01-0.1 \mathrm{pc}$ and a mass $\sim 1-10^{2} M_{\odot}$.
} 


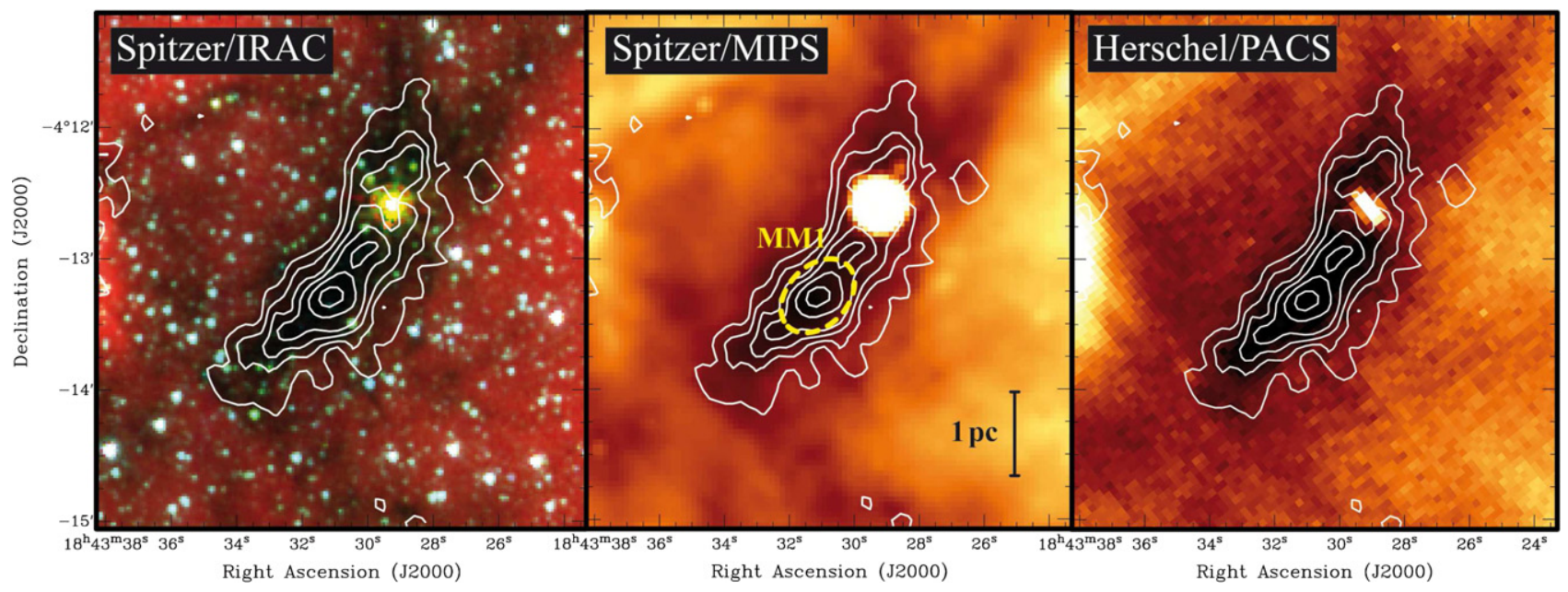

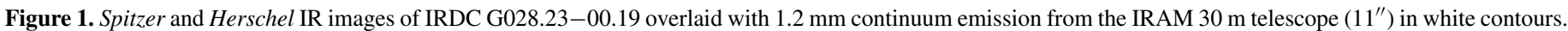

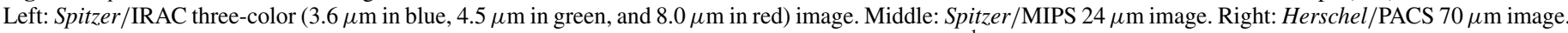

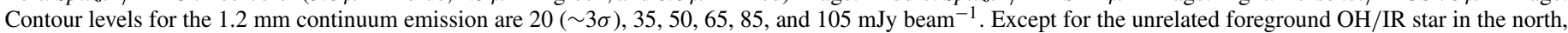
there is no IR evidence of embedded active star formation.

most elusive and earliest phase of high-mass star formation, the "prestellar" or "starless" phase.

Although several studies have focused on IRDCs, the number of studies on starless candidates is small (e.g., Pillai et al. 2011). Zhang et al. (2009) studied in detail an IR quiescent clump, apparently starless, resolving it into five cores at $1.3 \mathrm{~mm}$ with Submillimeter Array (SMA). However, new SMA observations at $0.88 \mathrm{~mm}$ obtained by Wang et al. (2011) show that all cores are associated with molecular outflows. This suggests that this clump is in fact forming stars which have entered the accretion phase. Thus, it is crucial to evaluate the prestellar nature of a core/clump by using interferometric observations of molecular lines, and their starless nature should not be asserted just by the lack of a detection in the IR.

Little is known about the chemistry of IRDCs. A few works have focused on molecular line surveys of several IRDCs (Sakai et al. 2008, 2010; Vasyunina et al. 2011; Sanhueza et al. 2012). For instance, Sanhueza et al. (2012) find chemical variations between different evolutionary stages using a set of 10 different molecular lines at $3.3 \mathrm{~mm}$. However, most of the effort related to chemistry in IRDCs has been focused on deuteration. Recent findings suggest that the deuterium fraction in high-mass starforming regions resembles that seen in low-mass star-forming regions after the stars turn on, decreasing with time as the clumps evolve to warmer temperatures (Chen et al. 2011; Miettinen et al. 2011; Fontani et al. 2011; Sakai et al. 2012; Pineda \& Teixeira 2013).

The internal kinematic structure of IRDCs has not yet been explored in depth. In two examples, IRDC G019.30+0.07 (Devine et al. 2011) and IRDC G035.39-00.33 (Jiménez-Serra et al. 2010; Henshaw et al. 2013), both IRDCs are composed of a few slightly different velocity components or "subclouds." It is still unclear what role these subclouds play in the earliest stages of high-mass star formation.

From the studies of Rathborne et al. (2010) and Sanhueza et al. (2012), we have identified an excellent candidate to study the initial conditions of massive star formation. As seen in Figure 1, IRDC G028.23-00.19 appears to be in a very early stage of evolution because it is dark at Spitzer/IRAC 3.6, 4.5, and $8.0 \mu \mathrm{m}$ (Benjamin et al. 2003), Spitzer/MIPS $24 \mu \mathrm{m}$ (Carey et al. 2009), and Herschel/PACS $70 \mu \mathrm{m}$ (Molinari et al. 2010), except for a bright unrelated IR source superimposed against the northern part of the cloud. This source is an OH/IR star (Bowers \& Knapp 1989), and is not a protostar. This foreground low-mass star, in a very late stage of evolution, is not associated with the IRDC. Indeed, its $V_{\mathrm{lsr}}$ is $\sim 52 \mathrm{~km} \mathrm{~s}^{-1}$, whereas for IRDC G028.23-00.19 it is $80 \mathrm{~km} \mathrm{~s}^{-1}$. Located in the center of the IRDC, the clump MM1 is one of the most massive, IR-quiescent clumps known, with a mass of $\sim 640 M_{\odot}$, as determined from $1.2 \mathrm{~mm}$ continuum emission (Rathborne et al. 2010) at a kinematical distance of $5.1 \mathrm{kpc}$ (Sanhueza et al. 2012). By fitting the spectral energy distribution (SED), Rathborne et al. (2010) determined for MM1 a dust temperature of $22 \mathrm{~K}$ (see Section 4.1 for the updated values of the mass and the temperature of MM1). Using the Mopra telescope (38"), Sanhueza et al. (2012) detected weak molecular line emission of $\mathrm{N}_{2} \mathrm{H}^{+}$, $\mathrm{HNC}$, and $\mathrm{HCO}^{+} J=1 \rightarrow 0$ toward this clump, consistent with cold gas. Battersby et al. (2010) observed this IRDC in $3.6 \mathrm{~cm}$ continuum emission with the Very Large Array (VLA) at $2^{\prime \prime}-3^{\prime \prime}$ angular resolution and found no $\mathrm{H}$ II regions associated with the cloud at $\sigma_{\text {rms }}=0.01 \mathrm{mJy}_{\text {beam }}{ }^{-1}$, which corresponds to a spectral type of B3 or later (Jackson \& Kraemer 1999). Chambers et al. (2009) searched for, but failed to detect, $22.23 \mathrm{GHz} \mathrm{H}_{2} \mathrm{O}$ and Class I $24.96 \mathrm{GHz} \mathrm{CH} \mathrm{CH}_{3} \mathrm{OH}$ masers with the Green Bank Telescope at $\sim 32^{\prime \prime}\left(\sigma_{\text {rms }} \cong 0.05 \mathrm{~K}\right)$. Based on the results obtained from a large variety of observations, we suggest that IRDC G028.23-00.19 harbors the prime example of a starless or prestellar massive clump (MM1), being in a very early stage of evolution and showing no evidence of active star formation.

To probe the chemistry, the internal structure, and the kinematics of IRDCs, interferometric observations are critical. In this paper, we present observations of IRDC G028.23-00.19 in nine different molecular lines obtained with the Combined Array for Research in Millimeter-wave Astronomy (CARMA). We aim to study the chemistry and internal structure of a massive star-forming region in a very early stage of evolution and test the starless nature of the cloud and, in particular, the clump MM1. This is the first study that explores the behavior of $\mathrm{NH}_{2} \mathrm{D}$, $\mathrm{H}^{13} \mathrm{CO}^{+}, \mathrm{SiO}, \mathrm{HN}^{13} \mathrm{C}, \mathrm{C}_{2} \mathrm{H}, \mathrm{HCO}^{+}, \mathrm{HNC}, \mathrm{N}_{2} \mathrm{H}^{+}$, and $\mathrm{CH}_{3} \mathrm{OH}$ at $\sim 11^{\prime \prime}$ angular resolution, studying both the chemistry and the structure of multiple subclouds traced by distinct, close velocity components $\left(\lesssim 2 \mathrm{~km} \mathrm{~s}^{-1}\right)$ in a massive star-forming region that, apparently, has not yet formed high-mass stars. 
Table 1

Summary of Observed Molecular Lines and Continuum Emission

\begin{tabular}{|c|c|c|c|c|c|c|c|}
\hline \multirow{2}{*}{$\begin{array}{l}\text { Molecule/ } \\
\text { Continuum }\end{array}$} & \multirow{2}{*}{$\begin{array}{l}\text { Molecular } \\
\text { Transition }\end{array}$} & \multirow{2}{*}{$\begin{array}{c}\text { Frequency } \\
(\mathrm{GHz})\end{array}$} & \multirow{2}{*}{$\begin{array}{c}E_{u} / k \\
(\mathrm{~K})\end{array}$} & \multirow{2}{*}{$\begin{array}{c}\text { Beam Size } \\
\left({ }^{\prime \prime} \times{ }^{\prime \prime}\right)\end{array}$} & \multirow{2}{*}{$\begin{array}{l}\text { P.A. } \\
\text { (deg) }\end{array}$} & \multicolumn{2}{|c|}{ rms Noise } \\
\hline & & & & & & $\left(\mathrm{mJy}\right.$ beam $\left.^{-1}\right)$ & $(\mathrm{mK})$ \\
\hline $\mathrm{NH}_{2} \mathrm{D}$ & $J_{K_{a}, K_{c}}=1_{1,1} \rightarrow 1_{0,1}$ & 85.926260 & 20.68 & $15.3 \times 8.4$ & -25.7 & 33.6 & 43.0 \\
\hline $\mathrm{H}^{13} \mathrm{CO}^{+}$ & $J=1 \rightarrow 0$ & 86.754330 & 4.16 & $15.0 \times 8.5$ & -26.9 & 31.1 & 39.8 \\
\hline $\mathrm{SiO}$ & $J=2 \rightarrow 1$ & 86.846998 & 6.25 & $15.0 \times 8.4$ & -27.0 & 31.0 & 39.7 \\
\hline $\mathrm{HN}^{13} \mathrm{C}$ & $J=1 \rightarrow 0$ & 87.090859 & 4.18 & $14.5 \times 8.6$ & -26.1 & 37.2 & 48.4 \\
\hline $\mathrm{C}_{2} \mathrm{H}$ & $N J F=1(3 / 2) 2 \rightarrow 0(1 / 2) 1$ & 87.316925 & 4.19 & $14.6 \times 8.6$ & -26.3 & 36.4 & 46.6 \\
\hline $\mathrm{HCO}^{+}$ & $J=1 \rightarrow 0$ & 89.188526 & 4.28 & $14.6 \times 8.2$ & -27.5 & 33.3 & 42.9 \\
\hline $\mathrm{HNC}$ & $J=1 \rightarrow 0$ & 90.663574 & 4.35 & $14.4 \times 8.0$ & -28.2 & 38.6 & 49.8 \\
\hline \multirow[t]{3}{*}{$\mathrm{N}_{2} \mathrm{H}^{+}$} & $J F_{1} F=112 \rightarrow 012$ & 93.171913 & 4.47 & $14.0 \times 7.8$ & -28.2 & 40.5 & 52.7 \\
\hline & $J F_{1} F=123 \rightarrow 012$ & 93.173772 & 4.47 & $14.0 \times 7.8$ & -28.2 & 40.5 & 52.7 \\
\hline & $J F_{1} F=101 \rightarrow 012$ & 93.176261 & 4.47 & $14.0 \times 7.8$ & -28.2 & 40.5 & 52.7 \\
\hline \multirow[t]{3}{*}{$\mathrm{CH}_{3} \mathrm{OH}$} & $J_{K}=2_{-1} \rightarrow 1_{-1} E$ & 96.739362 & 12.55 & $13.0 \times 7.6$ & -26.0 & 44.5 & 58.8 \\
\hline & $J_{K}=2_{0} \rightarrow 1_{0} A$ & 96.741375 & 6.97 & $13.0 \times 7.6$ & -26.0 & 44.5 & 58.8 \\
\hline & $J_{K}=2_{0} \rightarrow 1_{0} E$ & 96.744550 & 20.10 & $13.0 \times 7.6$ & -26.0 & 44.5 & 58.8 \\
\hline $3.3 \mathrm{~mm}$ & $\ldots$ & 91.877525 & $\ldots$ & $14.2 \times 7.9$ & -27.9 & 0.4 & $\ldots$ \\
\hline
\end{tabular}

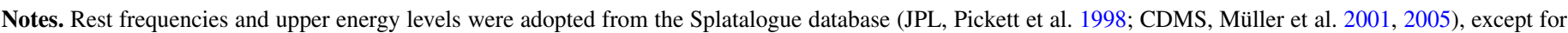
$\mathrm{N}_{2} \mathrm{H}^{+}$frequencies, which are from Daniel et al. (2006).

\section{OBSERVATIONS}

Observations were carried out with CARMA, a 15-element interferometer consisting of nine $6.1 \mathrm{~m}$ and six $10.4 \mathrm{~m}$ antennas, during the CARMA Summer School 2011 July 27 in the compact E configuration. The projected baselines range from 8 to $66 \mathrm{~m}$. The CARMA correlator has eight bands, each with an upper and lower sideband. One band was configured to have a bandwidth of $487.5 \mathrm{MHz}$, resulting in a total bandwidth of $\sim 1 \mathrm{GHz}$ by including both sidebands, to observe continuum emission at $3.3 \mathrm{~mm}$ for calibration $(91.878 \mathrm{GHz})$. The remaining seven bands were used to observe nine different molecular species $\left(\mathrm{NH}_{2} \mathrm{D}, \mathrm{H}^{13} \mathrm{CO}^{+}, \mathrm{SiO}, \mathrm{HN}^{13} \mathrm{C}, \mathrm{C}_{2} \mathrm{H}, \mathrm{HCO}^{+}\right.$, and $\mathrm{HNC}$ in the lower sidebands; and $\mathrm{N}_{2} \mathrm{H}^{+}$and $\mathrm{CH}_{3} \mathrm{OH}$ in the upper sidebands). For all seven bands, a bandwidth of $31 \mathrm{MHz}\left(\sim 100 \mathrm{~km} \mathrm{~s}^{-1}\right)$ and a spectral resolution of $97 \mathrm{kHz}$ $\left(\sim 0.3 \mathrm{~km} \mathrm{~s}^{-1}\right)$ were used. Using natural weighting, the $1 \sigma$ rms noise is 0.4 mJy beam $^{-1}$ for continuum emission, and $\sim 36.2 \mathrm{mJy}$ beam $^{-1}(\sim 46.9 \mathrm{mK})$ per channel for molecular lines. The average of the major and minor axis of the final synthesized beam was $10^{\prime} .9$, which corresponds to a physical size of $\sim 0.27 \mathrm{pc}$ at the distance of $5.1 \mathrm{kpc}$. The typical rms noise, synthesized beam, and position angle (P.A.) for the continuum emission and each molecular line are given in Table 1.

The system temperature varied from 190 to $250 \mathrm{~K}$ during the $7.1 \mathrm{hr}$ track (5.4 hr on source). The receivers were tuned to a local oscillator frequency of $91.918 \mathrm{GHz}$. The phase center of the observations is (R.A., decl. $)_{\mathrm{J} 2000}=\left(18^{\mathrm{h}} 43^{\mathrm{m}} 30^{\mathrm{s}} .78\right.$, $\left.-04^{\circ} 13^{\prime} 16^{\prime \prime} 25\right)$. At the center frequency of $91.918 \mathrm{GHz}$, the primary beams or fields of view of the 6.1 and $10.4 \mathrm{~m}$ antennas are $135^{\prime \prime}$ and $79^{\prime \prime}$, respectively.

The data were reduced and imaged in a standard way using MIRIAD software. We used the quasar 1743-038 as a phase calibrator, the quasar 1751+096 as a bandpass calibrator, and Neptune as a flux calibrator. The uncertainty in the absolute flux is about $15 \%$.

\section{RESULTS}

Figure 1 shows the Spitzer/IRAC 3.6, 4.5, and $8.0 \mu \mathrm{m}$ image (left), Spitzer/MIPS $24 \mu \mathrm{m}$ image (middle), and Herschel/ PACS $70 \mu \mathrm{m}$ image (right) overlaid with contours of the 1.2 continuum emission (11" angular resolution). As can be seen from the IR images, the IRDC is completely dark, except for the foreground $\mathrm{OH} / \mathrm{IR}$ star superimposed against the northern part of the cloud. The $1.2 \mathrm{~mm}$ continuum emission, from the IRAM $30 \mathrm{~m}$ telescope (Rathborne et al. 2006), matches the morphology of the IR extinction well. The massive clump MM1 is located at the center of the cloud.

Figure 2 shows the $3.3 \mathrm{~mm}$ continuum emission and integrated intensity maps of $\mathrm{N}_{2} \mathrm{H}^{+} J=1 \rightarrow 0, \mathrm{H}^{13} \mathrm{CO}^{+} J=1 \rightarrow 0$, and $\mathrm{HN}^{13} \mathrm{C} J=1 \rightarrow 0$ in images and color contours (ranges of integration are given in the figure caption). For $\mathrm{N}_{2} \mathrm{H}^{+}$, the transition with the lowest optical depth, the isolated hyperfine component $J F_{1} F=101 \rightarrow 012$ was used. White contours are the $1.2 \mathrm{~mm}$ continuum emission from the IRAM telescope. The $3.3 \mathrm{~mm}$ continuum emission from CARMA is more compact than the $1.2 \mathrm{~mm}$ emission. This is probably due to the low $3.3 \mathrm{~mm}$ flux at $12 \mathrm{~K}$ (see Section 4.1), the low sensitivity arising from using only $\sim 1 \mathrm{GHz}$ of continuum bandwidth, and because the interferometer may be missing extended flux. In fact, comparing the measured flux at $3.3 \mathrm{~mm}$ with the expected $3.3 \mathrm{~mm}$ flux from the SED fitting done in Section 4.1, we estimate that the interferometer is only recovering about $40 \%$ of the total flux. The emission of $\mathrm{N}_{2} \mathrm{H}^{+}, \mathrm{H}^{13} \mathrm{CO}^{+}$, and $\mathrm{HN}^{13} \mathrm{C}$ resembles the $1.2 \mathrm{~mm}$ emission from IRAM, although less extended. Using Mopra single-pointing observations from Sanhueza et al. (2012), we estimate that we recover all the flux for $\mathrm{N}_{2} \mathrm{H}^{+}$. Except for $\mathrm{N}_{2} \mathrm{H}^{+}, \mathrm{HCO}^{+}$, and $\mathrm{HNC}$, we have no observations from single-dish telescopes to evaluate if any other molecular line suffers from missing extended flux. Based on the spatial extent of $\mathrm{H}^{13} \mathrm{CO}^{+}, \mathrm{HN}^{13} \mathrm{C}, \mathrm{NH}_{2} \mathrm{D}, \mathrm{C}_{2} \mathrm{H}, \mathrm{SiO}$, and $\mathrm{CH}_{3} \mathrm{OH}$ emission, we suggest that zero-spacing problems are not important in these line observations and we recover most of the flux with the interferometer. On the other hand, using the Mopra single-pointing observations from Sanhueza et al. (2012), we estimate that $\sim 30 \%$ of the $\mathrm{HCO}^{+}$and $\mathrm{HNC}$ flux is recovered by the interferometer. $\mathrm{HCO}^{+}$and $\mathrm{HNC}$ integrated intensity maps are not presented here because their emission is substantially resolved out. Therefore, the distribution of these molecules is not discussed in the paper.

Figure 3 shows the integrated intensity maps of $\mathrm{NH}_{2} \mathrm{D}$ $J=1 \rightarrow 1, \mathrm{C}_{2} \mathrm{H} J=1 \rightarrow 0, \mathrm{SiO} J=2 \rightarrow 1$, and $\mathrm{CH}_{3} \mathrm{OH}$ $J=2 \rightarrow 1$ in images and color contours (ranges of integration are given in the figure caption). For $\mathrm{NH}_{2} \mathrm{D}$, the main component 


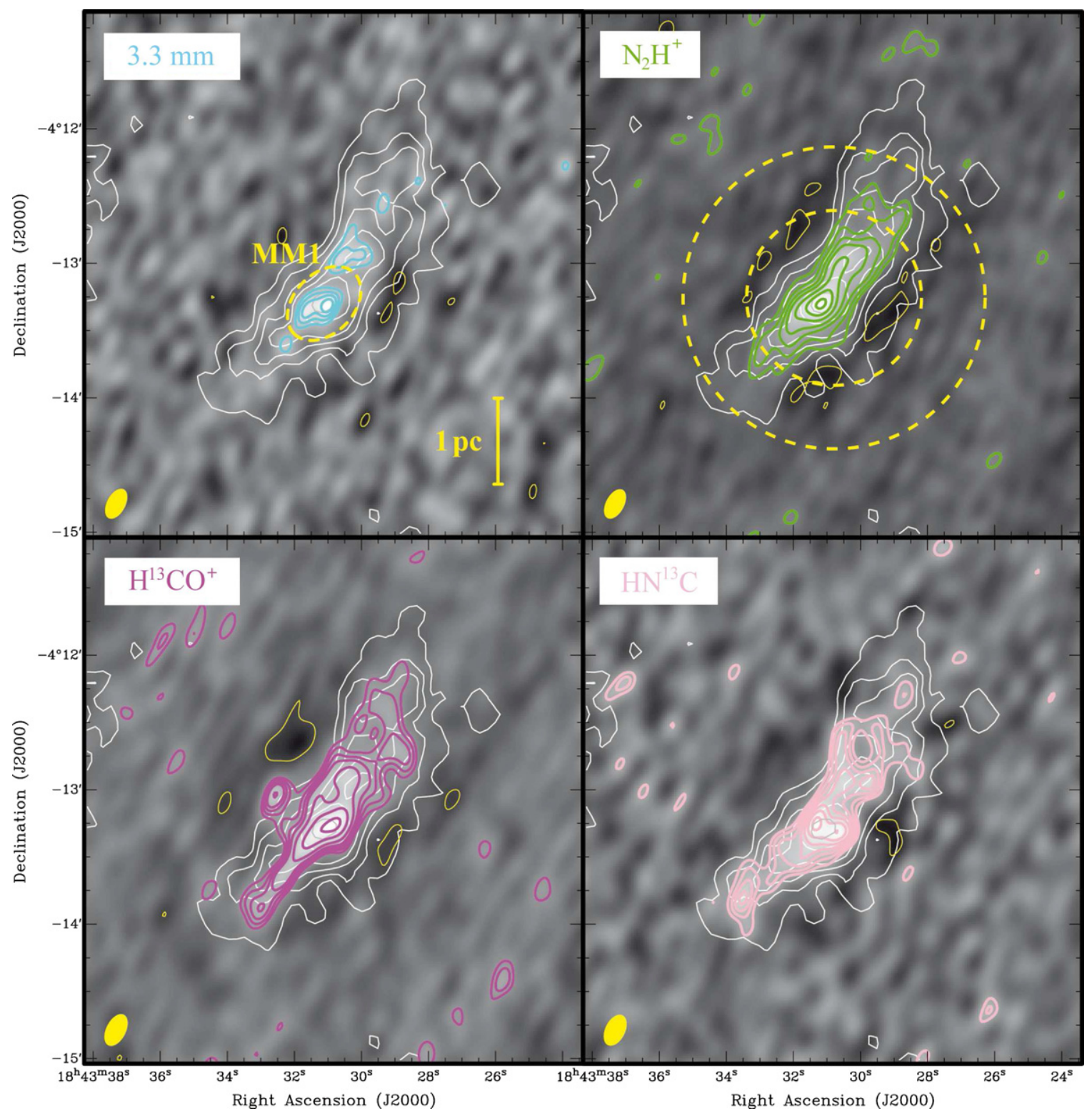

Figure 2. $\mathrm{N}_{2} \mathrm{H}^{+} J F_{1} F=101 \rightarrow 012, \mathrm{H}^{13} \mathrm{CO}^{+} J=1 \rightarrow 0$, and $\mathrm{HN}^{13} \mathrm{C} J=1 \rightarrow 0$ integrated intensity and $3.3 \mathrm{~mm}$ continuum emission maps of IRDC G028.23-00.19 in images and color contours. White contours correspond to the $1.2 \mathrm{~mm}$ continuum emission from the IRAM $30 \mathrm{~m}$ telescope (11"). Top left: contour levels for the $3.3 \mathrm{~mm}$ continuum emission are $-3 \sigma, 3 \sigma, 4 \sigma, 5 \sigma$, and $6 \sigma\left(\sigma=0.4 \mathrm{mJy}^{-1}\right.$ beam $\left.^{-1}\right)$. Top right: contour levels for the integrated intensity map of $\mathrm{N}_{2} \mathrm{H}^{+} J F_{1} F=101 \rightarrow 012$, between 76.9 and $82.8 \mathrm{~km} \mathrm{~s}^{-1}$, are $-4 \sigma, 3 \sigma, 5 \sigma, 7 \sigma, 9 \sigma, 12 \sigma, 15 \sigma$, and $18 \sigma\left(\sigma=54.0 \mathrm{mJy} \mathrm{beam}^{-1} \mathrm{~km} \mathrm{~s}^{-1}\right)$. Bottom left: contour levels for the integrated intensity map of $\mathrm{H}^{13} \mathrm{CO}^{+}$, between 76.6 and $82.4 \mathrm{~km} \mathrm{~s}^{-1}$, are $-4 \sigma, 3 \sigma, 4 \sigma, 5 \sigma, 6 \sigma, 8 \sigma, 11 \sigma$, and $14 \sigma\left(\sigma=43.3 \mathrm{mJy} \mathrm{beam}^{-1} \mathrm{~km} \mathrm{~s}^{-1}\right)$. Bottom right: contour levels for the integrated intensity map of $\mathrm{HN}^{13} \mathrm{C}$, between 77.0 and $82.7 \mathrm{~km} \mathrm{~s}^{-1}$, are $-4 \sigma, 3 \sigma, 4 \sigma, 5 \sigma, 6 \sigma, 7 \sigma, 8 \sigma$, and $9 \sigma\left(\sigma=51.6 \mathrm{mJy}\right.$ beam $\left.{ }^{-1} \mathrm{~km} \mathrm{~s}^{-1}\right)$. Contour levels for the $1.2 \mathrm{~mm}$ continuum emission are $20(\sim 3 \sigma), 35,50,65,85$, and $105 \mathrm{mJy}_{\text {beam }}{ }^{-1}$. Yellow dashed circles in top right panel show the primary beam of the 6.1 and $10.4 \mathrm{~m}$ antennas (135" and 79", respectively). The synthesized CARMA beam is shown in the bottom left of each panel.

$J_{K_{a}, K_{c}}=1_{1,1} \rightarrow 1_{0,1}$ was used. A velocity range including all three transitions was used for $\mathrm{CH}_{3} \mathrm{OH}$. The emission of $\mathrm{NH}_{2} \mathrm{D}$ and $\mathrm{C}_{2} \mathrm{H}$ is compact and is mostly located in the densest part of the IRDC, the MM1 clump. As discussed in Section 4.4, the enhancement of these molecules is likely due to cold prestellar gas-phase chemistry. Emission from $\mathrm{SiO}$ and $\mathrm{CH}_{3} \mathrm{OH}$ is highly unexpected because of the absence of IR signs of star formation (as discussed in more depth in Section 4.3). The $\mathrm{SiO}$ emission is located in the northern and southern regions of the IRDC, and it seems to be unassociated with the MM1 clump. An additional weak $\mathrm{SiO}$ component is located in the center-west part of the cloud. The $\mathrm{CH}_{3} \mathrm{OH}$ emission is widespread over the full IRDC.

Line center velocities, line widths, and intensity of the lines were determined by fitting Gaussian profiles. Molecu- lar lines that show hyperfine structures were fitted using a multi-Gaussian function, with fixed frequency separation between hyperfine transitions. Gaussian fit parameters of molecular lines, in selected positions, are shown in Table 2.

Multiple velocity components along a line of sight are not unusual in star-forming regions. However, it is not easy to separate two velocity components with a small offset of $\lesssim 2 \mathrm{~km} \mathrm{~s}^{-1}$ in massive star-forming regions. In general, these kind of regions have line widths of 3-4 $\mathrm{km} \mathrm{s}^{-1}$ (e.g., Hoq et al. 2013). Two close velocity components can be separated only when the line emission has narrow profiles and both components are not fully blended. This can be done in regions containing quiescent gas and using high-density tracers. Following the discussion of Devine et al. (2011), we will refer to the structures defined by 


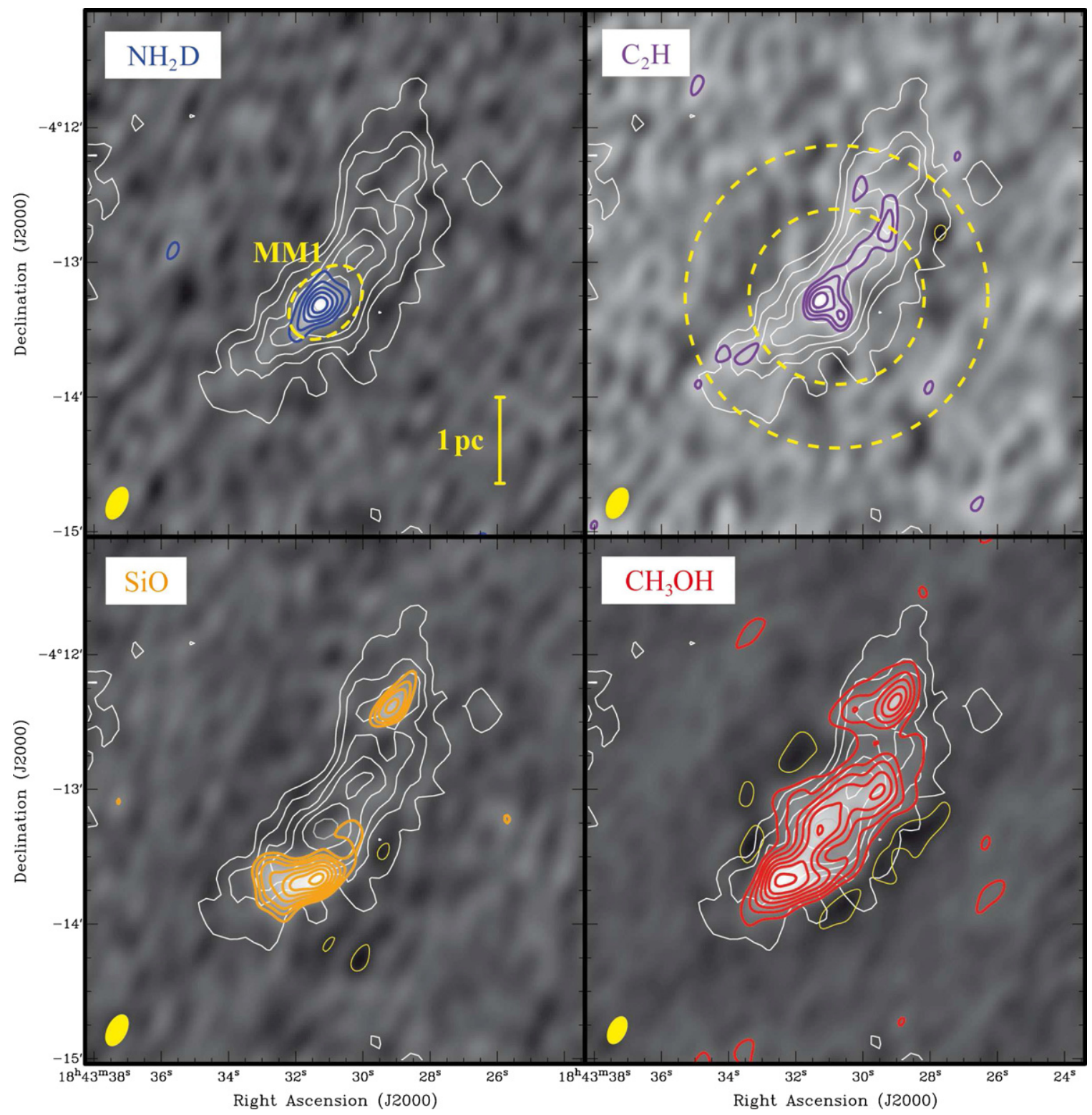

Figure 3. $\mathrm{NH}_{2} \mathrm{D} J=1 \rightarrow 1, \mathrm{C}_{2} \mathrm{H} J=1 \rightarrow 0, \mathrm{SiO} J=2 \rightarrow 1$, and $\mathrm{CH}_{3} \mathrm{OH} J=2 \rightarrow 1$ integrated intensity maps of IRDC G028.23-00.19 in images and color contours. White contours correspond to the $1.2 \mathrm{~mm}$ continuum emission from the IRAM $30 \mathrm{~m}$ telescope (11"). Top left: contour levels for the integrated intensity map of $\mathrm{NH}_{2} \mathrm{D}$, between 78.2 and $80.9 \mathrm{~km} \mathrm{~s}^{-1}$, are $-4 \sigma, 3 \sigma, 5 \sigma, 7 \sigma, 9 \sigma$, and $11 \sigma\left(\sigma=32.3 \mathrm{mJy}_{\text {beam }}^{-1} \mathrm{~km} \mathrm{~s}^{-1}\right)$. Top right: contour levels for the integrated intensity map of $\mathrm{C}_{2} \mathrm{H}$, between 77.7 and $82.7 \mathrm{~km} \mathrm{~s}^{-1}$, are $-4 \sigma, 3 \sigma, 4 \sigma, 5 \sigma$, and $6 \sigma\left(\sigma=47.3 \mathrm{mJy} \mathrm{beam}^{-1} \mathrm{~km} \mathrm{~s}^{-1}\right)$. Bottom left: contour levels for the integrated intensity map of $\mathrm{SiO}$, between 70.6 and $84.7 \mathrm{~km} \mathrm{~s}^{-1}$, are $-4 \sigma, 4 \sigma, 6 \sigma, 8 \sigma, 11 \sigma, 14 \sigma, 17 \sigma$, and $20 \sigma\left(\sigma=67.7 \mathrm{mJy}\right.$ beam $\left.^{-1} \mathrm{~km} \mathrm{~s}^{-1}\right)$. Bottom right: contour levels for the integrated intensity map of all three $\mathrm{CH}_{3} \mathrm{OH}$ transitions, between 72.9 and $90.8 \mathrm{~km} \mathrm{~s}^{-1}$, are $-5 \sigma, 4 \sigma, 9 \sigma, 14 \sigma, 20 \sigma, 26 \sigma, 32 \sigma$, and $40 \sigma\left(\sigma=103.6 \mathrm{mJy}^{\mathrm{b}}\right.$ beam $\left.{ }^{-1} \mathrm{~km} \mathrm{~s}^{-1}\right)$. Contour levels for the $1.2 \mathrm{~mm}$ continuum emission are $20(\sim 3 \sigma), 35,50,65,85$, and 105 mJy beam $^{-1}$. Yellow dashed circles in top right panel show the primary beam of the 6.1 and $10.4 \mathrm{~m}$ antennas (135" and 79", respectively). The synthesized CARMA beam is shown in the bottom left of each panel.

Table 2

Gaussian Fit Parameters of Molecular Lines in Selected Positions

\begin{tabular}{|c|c|c|c|c|c|}
\hline Molecule & Transition & $\begin{array}{l}T_{\mathrm{A}} \\
(\mathrm{K})\end{array}$ & $\begin{array}{c}V_{\mathrm{lsr}} \\
\left(\mathrm{km} \mathrm{s}^{-1}\right)\end{array}$ & $\begin{array}{c}\Delta V \\
\left(\mathrm{~km} \mathrm{~s}^{-1}\right)\end{array}$ & Position \\
\hline $\mathrm{NH}_{2} \mathrm{D}$ & $J_{K_{a}, K_{c}}=1_{1,1} \rightarrow 1_{0,1}$ & $0.36 \pm 0.03$ & $79.64 \pm 0.05$ & $1.34 \pm 0.13$ & MM1 \\
\hline $\mathrm{H}^{13} \mathrm{CO}^{+}$ & $J=1 \rightarrow 0$ & $0.28 \pm 0.02$ & $79.42 \pm 0.09$ & $2.41 \pm 0.21$ & MM1 \\
\hline $\mathrm{HN}^{13} \mathrm{C}$ & $J=1 \rightarrow 0$ & $0.28 \pm 0.03$ & $79.51 \pm 0.09$ & $1.89 \pm 0.21$ & MM1 \\
\hline $\mathrm{C}_{2} \mathrm{H}$ & $N J F=1(3 / 2) 2 \rightarrow 0(1 / 2) 1$ & $0.18 \pm 0.03$ & $79.28 \pm 0.15$ & $1.82 \pm 0.36$ & MM1 \\
\hline $\mathrm{N}_{2} \mathrm{H}^{+}$ & $J F_{1} F=101 \rightarrow 012$ & $0.66 \pm 0.03$ & $79.17 \pm 0.04$ & $1.85 \pm 0.10$ & MM1 \\
\hline \multirow[t]{3}{*}{$\mathrm{CH}_{3} \mathrm{OH}$} & $J_{K}=2_{-1} \rightarrow 1_{-1} E$ & $0.89 \pm 0.03$ & $79.32 \pm 0.02$ & $2.18 \pm 0.09$ & MM1 \\
\hline & $J_{K}=2_{0} \rightarrow 1_{0} A$ & $1.12 \pm 0.03$ & $79.32 \pm 0.02$ & $2.13 \pm 0.07$ & MM1 \\
\hline & $J_{K}=2_{0} \rightarrow 1_{0} E$ & $0.13 \pm 0.04$ & $79.32 \pm 0.02$ & $1.44 \pm 0.47$ & MM1 \\
\hline \multirow[t]{3}{*}{$\mathrm{SiO}$} & $J=2 \rightarrow 1$ & $0.14 \pm 0.02$ & $81.79 \pm 0.15$ & $1.94 \pm 0.34$ & Center-west \\
\hline & & $0.26 \pm 0.01$ & $76.31 \pm 0.15$ & $6.26 \pm 0.36$ & South \\
\hline & & $0.13 \pm 0.01$ & $80.48 \pm 0.33$ & $7.74 \pm 0.79$ & North \\
\hline
\end{tabular}



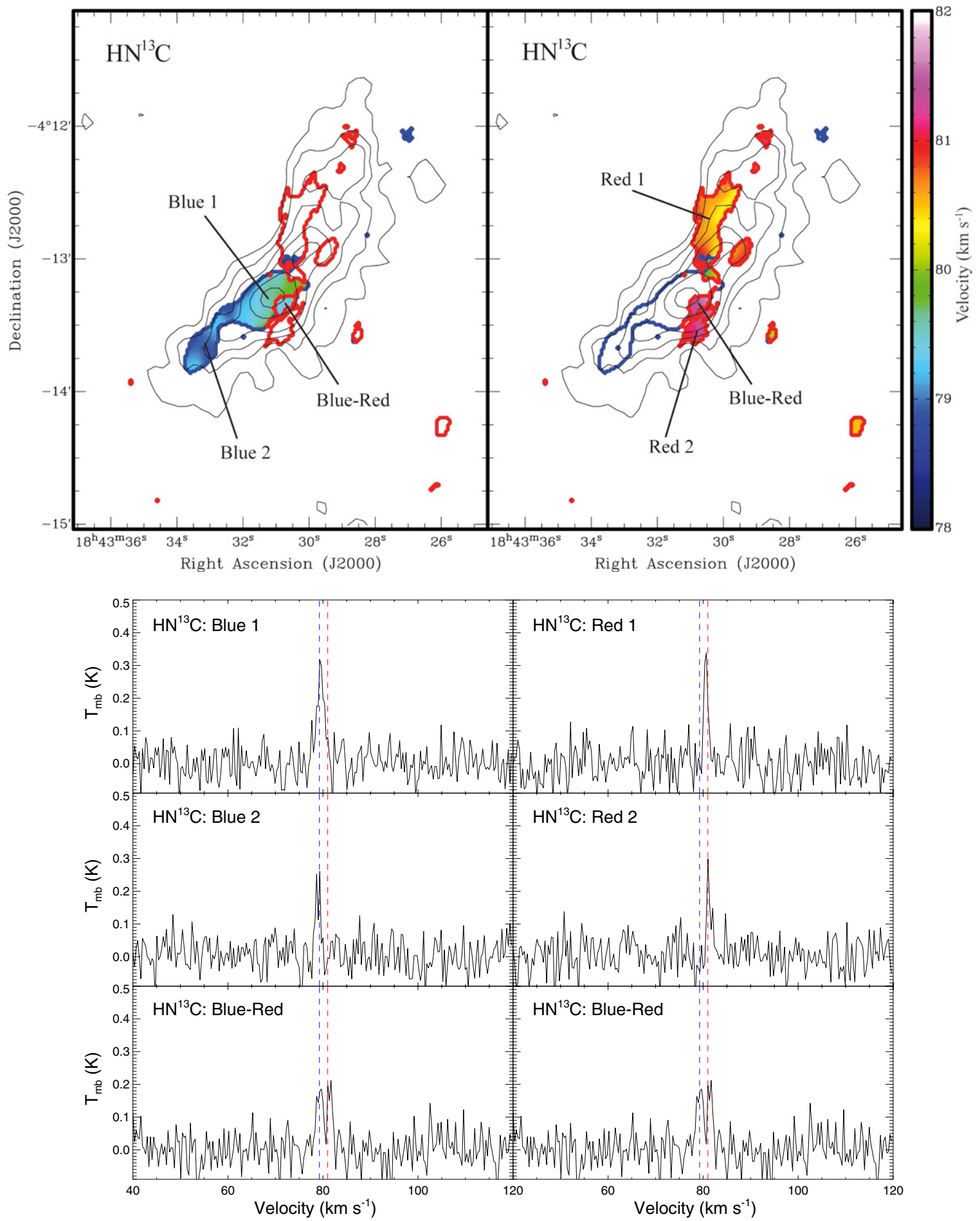

Figure 4. Top left: $\mathrm{HN}^{13} \mathrm{C} J=1 \rightarrow 0$ central velocity map of the blueshifted subcloud (average central velocity of $79.3 \mathrm{~km} \mathrm{~s}^{-1}$ ). Top right: $\mathrm{HN}{ }^{13} \mathrm{C} J=1 \rightarrow 0$ center velocity map of the redshifted subcloud (average central velocity of $80.7 \mathrm{~km} \mathrm{~s}^{-1}$ ). Black contours correspond to the $1.2 \mathrm{~mm}$ continuum emission from the IRAM $30 \mathrm{~m}$ telescope. Bottom: $\mathrm{HN}^{13} \mathrm{C} J=1 \rightarrow 0$ spectra in selected positions. Dashed blue and red lines show the average center velocity of the subclouds. Both spectra in the bottom show the same position in the cloud where the subclouds overlap.

close velocity components as "subclouds." $\mathrm{HN}^{13} \mathrm{C} J=1 \rightarrow 0$ and $\mathrm{C}_{2} \mathrm{H} J=1 \rightarrow 0$ show double-line profiles in the centerwest part of the IRDC. The double-line profiles are actually two close velocity components separated by $\sim 2 \mathrm{~km} \mathrm{~s}^{-1}$. Each individual component can be traced continuously from the overlapping region of the subclouds, where we see the double-line profiles, to regions where only one of the components is seen. Two velocity components are also seen in $\mathrm{H}^{13} \mathrm{CO}^{+}, \mathrm{N}_{2} \mathrm{H}^{+}$, and
$\mathrm{CH}_{3} \mathrm{OH}$; however, because these molecules have broader line emission, both components are blended. Figure 4 shows the $\mathrm{HN}^{13} \mathrm{C}$ center velocity map of each component obtained by fitting Gaussian profiles to the spectra. The map shows two spatially coherent structures. The blueshifted subcloud is mostly located in the southern-central part of the IRDC and has an average central velocity of $79.3 \mathrm{~km} \mathrm{~s}^{-1}$. The redshifted subcloud is mostly located in the northern-central region of the 
IRDC and has an average central velocity of $80.7 \mathrm{~km} \mathrm{~s}^{-1}$. The velocity difference between subclouds is therefore $\sim 1.4 \mathrm{~km} \mathrm{~s}^{-1}$, similar to those found in other IRDCs, such as G019.30+0.07 or G035.39-00.33 ( $<2 \mathrm{~km} \mathrm{~s}^{-1}$; see Devine et al. 2011; Henshaw et al. 2013). The massive MM1 clump is associated with the blueshifted subcloud. $\mathrm{NH}_{2} \mathrm{D}$ emission is only detected in the blueshifted subcloud, while the narrow $\mathrm{SiO}$ component (described in the next paragraph) is only detected in the redshifted subcloud. All other lines are detected in both substructures.

Figure 5 shows line width and center velocity maps of $\mathrm{SiO}$ across the full IRDC and spectra of $\mathrm{SiO}$ and $\mathrm{CH}_{3} \mathrm{OH}$ in selected positions. Because in several positions over the IRDC the $\mathrm{CH}_{3} \mathrm{OH}$ transitions are blended, Gaussian fits cannot be performed for all methanol spectra and thus the line width and center velocity maps are not useful. The line width map of the $\mathrm{SiO}$ line reveals the presence of two distinct $\mathrm{SiO}$ components defined by having different line widths and being located in different positions. The first $\mathrm{SiO}$ component has narrow line widths, $\sim 2 \mathrm{~km} \mathrm{~s}^{-1}$, and peaks at the redshifted velocity component. It is also located in the center-west part of the IRDC, at about the same position where the subclouds overlap (see right panel in Figure 5). The second $\mathrm{SiO}$ component has broader line widths, $\gtrsim 6 \mathrm{~km} \mathrm{~s}^{-1}$, and is located in the southern and northern regions of the IRDC. $\mathrm{CH}_{3} \mathrm{OH}$ emission is also broad in the same positions where $\mathrm{SiO}$ displays broad line profiles. Figure 5 also displays the $\mathrm{SiO}$ and $\mathrm{CH}_{3} \mathrm{OH}$ spectra at the center position of MM1. As expected for quiescent molecular gas (Martin-Pintado et al. 1992; Requena-Torres et al. 2007), the $\mathrm{SiO}$ emission is not detected at this position. The $\mathrm{CH}_{3} \mathrm{OH}$ $J_{K}=2_{-1} \rightarrow 1_{-1} E$ and $J_{K}=2_{0} \rightarrow 1_{0} A$ transitions $\left(E_{u} / k\right.$ of 12.6 and $7.0 \mathrm{~K}$, respectively) are bright and are not blended. The higher-excitation $\mathrm{CH}_{3} \mathrm{OH} J_{K}=2_{0} \rightarrow 1_{0} E$ transition $\left(E_{u} / k=20.1 \mathrm{~K}\right)$ is barely detected at $3 \sigma$ level.

Figure 6 shows all lines at the peak position of $\mathrm{NH}_{2} \mathrm{D}$ (MM1). $\mathrm{CH}_{3} \mathrm{OH}$ is the brightest line at this position. $\mathrm{NH}_{2} \mathrm{D}, \mathrm{C}_{2} \mathrm{H}$, $\mathrm{HN}^{13} \mathrm{C}$, and $\mathrm{H}^{13} \mathrm{CO}^{+}$lines show narrow profiles. $\mathrm{SiO}$ emission is not detected. Because the $\mathrm{N}_{2} \mathrm{H}^{+} J F_{1} F=123 \rightarrow 012$ and $J F_{1} F=112 \rightarrow 012$ transitions are saturated, the usually weakest $J F_{1} F=101 \rightarrow 012$ is the brightest line, suggesting a large optical depth for $\mathrm{N}_{2} \mathrm{H}^{+} J=1 \rightarrow 0$. $\mathrm{HCO}^{+}$and $\mathrm{HNC}$ show only faint emission in $\mathrm{MM} 1$. Both $\mathrm{HCO}^{+}$and $\mathrm{HNC}$ show profiles with absorption features that prevent their use in calculating physical parameters and make their interpretation difficult.

\section{DISCUSSION}

\subsection{Dust Temperature, $\mathrm{H}_{2}$ Column Density, and Mass Determination}

Taking advantage of the new Herschel Hi-GAL Survey (Molinari et al. 2010), we derive the dust temperature for MM1 by using the emission detected at 250,350 , and $500 \mu \mathrm{m}$, and the $1.2 \mathrm{~mm}$ emission from IRAM $30 \mathrm{~m}$ (Rathborne et al. 2006). We exclude from the analysis the 70 and $160 \mu \mathrm{m}$ emission maps. At $70 \mu \mathrm{m}$ the IRDC is seen in absorption and at $160 \mu \mathrm{m}$ the emission from the IRDC is indistinguishable from the background/foreground emission.

After convolving the $250 \mu \mathrm{m}, 350 \mu \mathrm{m}$, and $1.2 \mathrm{~mm}$ maps to the angular resolution of the $500 \mu \mathrm{m}$ map (35". 1$)$, we subtract an extended component from the Herschel maps that represents the contribution from the background and foreground diffuse emission. Then, we fit the fluxes measured at the peak position of MM1 by using a single-temperature emission model, assuming
Table 3

Derived Parameters in Selected Positions

\begin{tabular}{lcccc}
\hline \hline Molecule & $\begin{array}{c}N_{\text {Molecule }} \\
\left(\mathrm{cm}^{-2}\right)\end{array}$ & $\begin{array}{c}N_{\mathrm{H}_{2}} \\
\left(\mathrm{~cm}^{-2}\right)\end{array}$ & $\begin{array}{c}\text { Molecular } \\
\text { Abundance }\end{array}$ & Position \\
\hline $\mathrm{NH}_{2} \mathrm{D}$ & $5.2(2.0) \times 10^{13}$ & $3.9(1.4) \times 10^{22}$ & $1.3(0.7) \times 10^{-9}$ & MM1 \\
$\mathrm{HCO}^{+}$ & $4.5(0.6) \times 10^{13}$ & $3.9(1.4) \times 10^{22}$ & $1.2(0.5) \times 10^{-9}$ & MM1 \\
$\mathrm{HNC}$ & $5.7(0.9) \times 10^{13}$ & $3.9(1.4) \times 10^{22}$ & $1.5(0.6) \times 10^{-9}$ & MM1 \\
$\mathrm{C}_{2} \mathrm{H}$ & $1.6(0.4) \times 10^{13}$ & $3.9(1.4) \times 10^{22}$ & $4.1(1.8) \times 10^{-10}$ & MM1 \\
$\mathrm{N}_{2} \mathrm{H}^{+}$ & $1.7(0.2) \times 10^{13}$ & $3.9(1.4) \times 10^{22}$ & $4.3(1.6) \times 10^{-10}$ & MM1 \\
$\mathrm{CH}_{3} \mathrm{OH}$ & $1.1(0.1) \times 10^{14}$ & $3.9(1.4) \times 10^{22}$ & $2.7(1.0) \times 10^{-9}$ & MM1 \\
$\mathrm{SiO}$ & $6.7(1.6) \times 10^{11}$ & $1.6(0.5) \times 10^{22}$ & $4.3(1.8) \times 10^{-11}$ & Center-west \\
& $6.8(0.6) \times 10^{12}$ & $1.8(0.7) \times 10^{21}$ & $3.8(1.4) \times 10^{-9}$ & South \\
& $8.1(1.2) \times 10^{12}$ & $1.1(0.4) \times 10^{22}$ & $7.5(2.7) \times 10^{-10}$ & North \\
\hline
\end{tabular}

optically thin emission, and dust opacities $\left(\kappa_{v}\right)$ for dust grains with thin ice mantles coagulated at $10^{5} \mathrm{~cm}^{-3}$ from Ossenkopf $\&$ Henning (1994). We defer the detailed description of the extended emission subtraction algorithm and the fitting procedure to an upcoming paper (A. Guzmán et al., in preparation). Using the measured fluxes at $250 \mu \mathrm{m}\left(150.0 \mathrm{MJy} \mathrm{sr}^{-1}\right), 350 \mu \mathrm{m}$ (610.8 $\mathrm{MJy} \mathrm{sr}^{-1}$ ), $500 \mu \mathrm{m}$ (313.9 $\mathrm{MJy} \mathrm{sr}^{-1}$ ), and $1.2 \mathrm{~mm}$ (19.5 $\left.\mathrm{MJy} \mathrm{sr}^{-1}\right)$, we determine a dust temperature $\left(T_{\text {dust }}\right)$ of $12 \pm 2 \mathrm{~K}$. We suggest that the main difference with the temperature of $22 \mathrm{~K}$ determined by Rathborne et al. (2010) is that they use a low value for the dust emissivity index $(\beta=1)$ and use the potentially optically thick Spitzer 85 and $95 \mu \mathrm{m}$ emission in fitting the SED. The new dust temperature is more consistent with the $\mathrm{CH}_{3} \mathrm{OH}$ temperature we determine by using the rotational diagram technique in Section $4.2(12 \mathrm{~K})$ and the $\mathrm{NH}_{3}$ temperature $(8 \mathrm{~K})$ recently determined by Chira et al. (2013).

To calculate the $\mathrm{H}_{2}$ column density and mass from the $1.2 \mathrm{~mm}$ continuum, we used the same procedure of Rathborne et al. (2006), assuming a gas-to-dust mass ratio of 100 and adopting for the dust opacity $\left(\kappa_{1.2 \mathrm{~mm}}\right)$ a value of $1.0 \mathrm{~cm}^{2} \mathrm{~g}^{-1}$. Computed column densities at a given position are shown in Table 3. For the mass, we use the same integrated flux measure by Rathborne et al. (2010; $1.63 \mathrm{Jy})$ and the distance determined by Sanhueza et al. $(2012 ; 5.1 \mathrm{kpc})$. We obtain a mass of $1520 M_{\odot}$ for the clump MM1.

\subsection{Molecular Column Densities and Abundances}

Assuming local thermodynamic equilibrium (LTE) conditions, beam-averaged column densities of $\mathrm{SiO}, \mathrm{H}^{13} \mathrm{CO}^{+}, \mathrm{HN}^{13} \mathrm{C}$, $\mathrm{C}_{2} \mathrm{H}$, and $\mathrm{N}_{2} \mathrm{H}^{+}$were calculated using the description of Sanhueza et al. (2012). Sanhueza et al. (2012) obtain column densities for the same set of lines and list all parameters needed for the calculation. For this work, we have assumed that all lines listed above are optically thin. However, there is evidence that the $\mathrm{C}_{2} \mathrm{H}$ and $\mathrm{N}_{2} \mathrm{H}^{+}$emission lines may be moderately optically thick; their column densities should thus be treated as lower limits. $\mathrm{HCO}^{+}$and $\mathrm{HNC}$ column densities are calculated from $\mathrm{H}^{13} \mathrm{CO}^{+}$and $\mathrm{HN}^{13} \mathrm{C}$, adopting a $\left[{ }^{12} \mathrm{C} /{ }^{13} \mathrm{C}\right]$ isotopic abundance ratio of 50 (see discussion of Milam et al. 2005). Under LTE conditions, we assume an excitation temperature $\left(T_{\mathrm{ex}}\right)$ equal to the dust temperature determined in Section 4.1 (12 K).

For $\mathrm{NH}_{2} \mathrm{D}$, the optical depth of the main hyperfine component $\left(\tau_{\mathrm{m}}\right)$ was calculated from the ratio between the observed main beam brightness temperatures of the main component $\left(T_{\mathrm{mb}_{\mathrm{m}}}\right)$ and the brightest satellite $\left(T_{\mathrm{mb}_{\mathrm{s}}}\right)$, using the following relation (e.g., Sanhueza et al. 2012)

$$
\frac{1-e^{-\frac{1}{3} \tau_{\mathrm{m}}}}{1-e^{-\tau_{\mathrm{m}}}}=\frac{T_{\mathrm{mb}_{\mathrm{s}}}}{T_{\mathrm{mb}_{\mathrm{m}}}},
$$



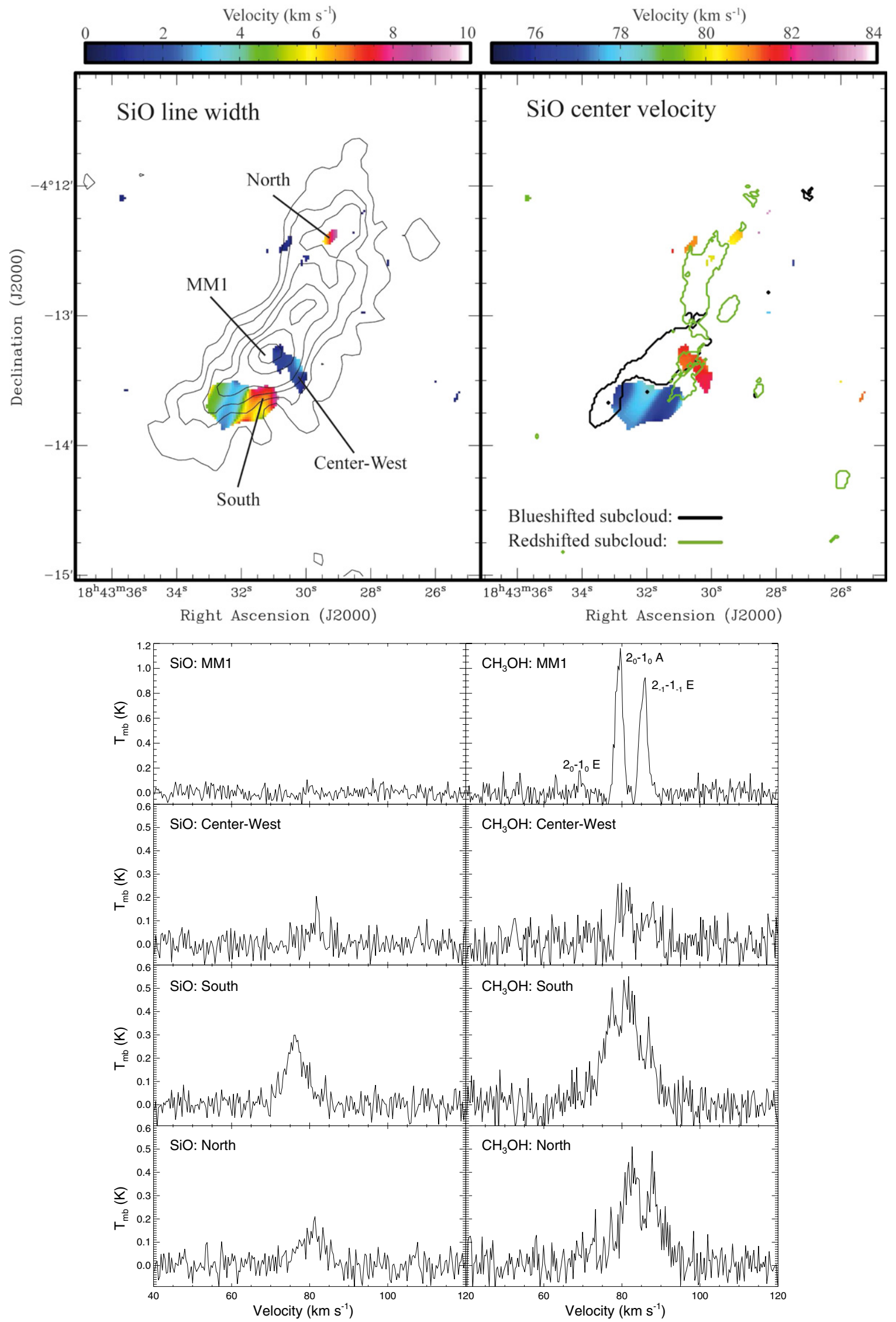

Figure 5. Top left: line width velocity map of $\mathrm{SiO} J=2 \rightarrow 1$. Top right: center velocity map of $\mathrm{SiO} J=2 \rightarrow 1$. Black contours correspond to the $1.2 \mathrm{~mm}$ continuum emission from the IRAM 30 m telescope. Bottom: spectra of $\mathrm{SiO}$ (left) and $\mathrm{CH}_{3} \mathrm{OH}$ (right) in selected positions. In MM1, there is no SiO $J=2 \rightarrow 1$ emission and $\mathrm{CH}_{3} \mathrm{OH} J=2 \rightarrow 1$ shows narrow Gaussian profiles. In the northern and southern regions of the IRDC, $\mathrm{SiO} J=2 \rightarrow 1$ and $\mathrm{CH}_{3} \mathrm{OH} J=2 \rightarrow 1$ lines have broad line widths $\left(\gtrsim 6 \mathrm{~km} \mathrm{~s}^{-1}\right)$. In the center-west part of the IRDC, SiO $J=2 \rightarrow 1$ lines show narrow $\left(\sim 2 \mathrm{~km} \mathrm{~s}^{-1}\right)$ and weak profiles. 


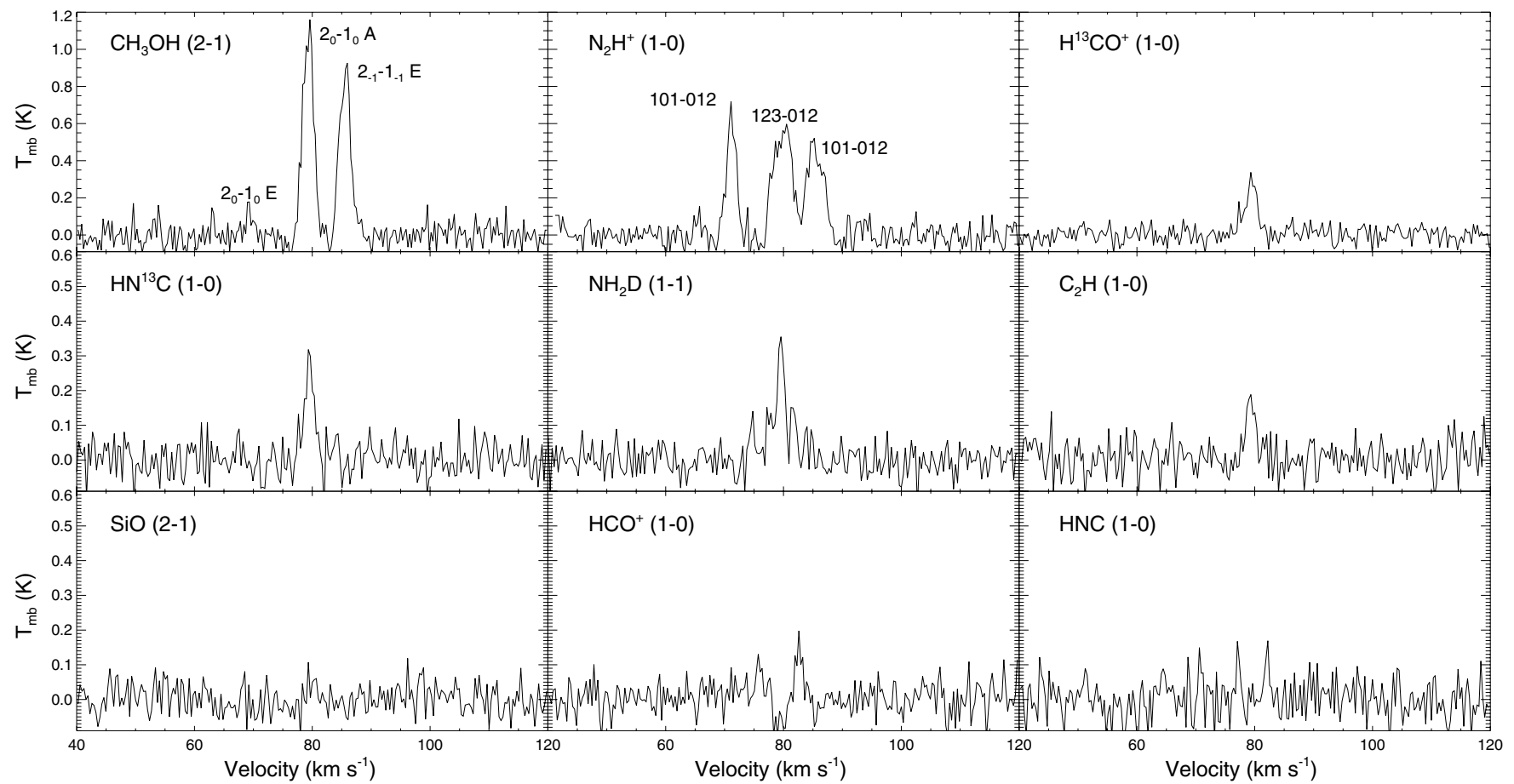

Figure 6. Spectra of all nine molecular lines at the peak position of $\mathrm{NH}_{2} \mathrm{D}$ (MM1). Two $\mathrm{CH}_{3} \mathrm{OH}$ transitions show the brightest emission. The $\mathrm{N}_{2} \mathrm{H}^{+} J F_{1} F=123 \rightarrow 012$ and $J F_{1} F=112 \rightarrow 012$ transitions are saturated, and the usually weakest $J F_{1} F=101 \rightarrow 012$ seems to be the brightest line. SiO is not detected at this position.

where we have used that the opacity ratio $(r)$ between the main ( $\left.\tau_{\mathrm{m}}\right)$ and satellite $\left(\tau_{\mathrm{s}}\right)$ lines is $r=\tau_{\mathrm{m}} / \tau_{\mathrm{s}}=3$, which depends only on the transition moments. We obtained an optical depth of 0.7 for the main hyperfine component.

In LTE conditions $\left(T_{\mathrm{ex}}=T_{\mathrm{dust}}\right)$, the column density is given by (e.g., Garden et al. 1991; Sanhueza et al. 2012)

$$
N=\frac{8 \pi v^{3}}{c^{3} R} \frac{Q_{\mathrm{rot}}}{g_{\mathrm{u}} A_{\mathrm{ul}}} \frac{\exp \left(E_{l} / k T_{\mathrm{ex}}\right)}{\left[1-\exp \left(-h v / k T_{\mathrm{ex}}\right)\right]} \int \tau d v
$$

where $\tau_{v}$ is the optical depth of the line, $g_{u}$ is the statistical weight of the upper level, $A_{\mathrm{ul}}$ is the Einstein coefficient for spontaneous emission, $E_{l}$ is the energy of the lower state, $Q_{\text {rot }}=3.899+0.751 T_{\mathrm{ex}}^{3 / 2}$ is the partition function for $\mathrm{NH}_{2} \mathrm{D}$ (Busquet et al. 2010), $v$ is the transition frequency, and $R=1 / 2$ is the relative intensity of the main hyperfine transition with respect to the others. $R$ is only relevant for hyperfine transitions because it takes into account the satellite lines correcting by their relative opacities. It is equal to 1.0 for transitions without hyperfine structure. The values of $g_{\mathrm{u}}, A_{\mathrm{ul}}$, and $E_{l}$ are given in Table 4 . The beam-averaged column density is obtained by multiplying $N$ by the filling factor, $f$. The filling factor can be found from

$$
T_{\mathrm{mb}}=f\left[J\left(T_{\mathrm{ex}}\right)-J\left(T_{\mathrm{bg}}\right)\right]\left(1-e^{-\tau_{v}}\right),
$$

where $J(T)$ is given by

$$
J(T)=\frac{h v}{k} \frac{1}{e^{h v / k T}-1} .
$$

The derived $\mathrm{NH}_{2} \mathrm{D}$ column density and filling factor are $6.2 \times 10^{14} \mathrm{~cm}^{-2}$ and 0.08 , resulting in a beam-averaged column density of $5.2 \times 10^{13} \mathrm{~cm}^{-2}$ in MM1.

The $\mathrm{CH}_{3} \mathrm{OH}$ column density $(N)$ was obtained by using the rotational diagram technique in the most general form (e.g.,
Girart et al. 2002). Assuming that all rotational levels are populated with the same excitation temperature (LTE), we have

$$
\log I=\log \frac{f N}{Q_{\text {rot }}}-\frac{E_{\mathrm{u}}}{T} \log e-\log C_{T}-\log F_{T},
$$

with $Q_{\text {rot }}=1.2327 T^{1.5}$ (the rotational partition function for $\left.\mathrm{CH}_{3} \mathrm{OH}\right)$ and $I$ equal to

$$
I=\frac{3 k \int T_{\mathrm{mb}} d v}{8 \pi^{3} g_{K} g_{I} S \mu^{2} v},
$$

where $g_{K}$ and $g_{I}$ are the $K$-level and nuclear spin degeneracies, $\mu$ is the electric dipole moment of the molecule, and $S$ is the line strength. $C_{T}$ and $F_{T}$ are defined as

$$
C_{T}=\frac{\tau}{1-e^{-\tau}} \quad \text { and } \quad F_{T}=\frac{J\left(T_{\mathrm{ex}}\right)}{J\left(T_{\mathrm{ex}}\right)-J\left(T_{\mathrm{bg}}\right)} .
$$

The optical depth of the line is given by

$$
\tau=\frac{8 \pi^{3} g_{K} g_{I} \mu^{2} S v}{3 h} \frac{N}{Q_{\mathrm{rot}} \Delta v}\left(e^{h v / k T}-1\right) e^{-E_{\mathrm{u}} / k T},
$$

where $E_{\mathrm{u}}$ is the energy of the upper state and $\Delta v$ is the line width. Values for $g_{K}, g_{I}$, and $\mu^{2} S$ are given in Table 4. A detailed description and derivation of the equations used in the rotational diagram technique are presented in A. Silva \& Q. Zhang (in preparation).

Equation (5) is frequently used without the $C_{T}$ and $F_{T}$ terms, assuming that $\tau \ll 1$ and $T_{\mathrm{ex}} \gg T_{\mathrm{bg}}$. However, in the cold clump MM1 these assumptions may not be valid. An additional condition for cold gas is that the $E / A$ abundance ratio is expected to be 0.69 at low temperatures $(\sim 10 \mathrm{~K})$. This is because the ground state of $E$-methanol is $7.9 \mathrm{~K}$ above the ground state of $A$-methanol, which results in an 
Table 4

Parameters Used for $\mathrm{NH}_{2} \mathrm{D}$ and $\mathrm{CH}_{3} \mathrm{OH}$ Column Density Calculations

\begin{tabular}{lccccccc}
\hline \hline Molecule & Transition & $g_{u}$ & $g_{K}$ & $g_{I}$ & $\begin{array}{c}A_{\mathrm{ul}} \\
\left(\times 10^{-6} \mathrm{~s}^{-1}\right)\end{array}$ & $\begin{array}{c}E_{l} / k \\
(\mathrm{~K})\end{array}$ & $\begin{array}{c}\mu^{2} S \\
\left(\mathrm{D}^{2}\right)\end{array}$ \\
\hline $\mathrm{NH}_{2} \mathrm{D}$ & $J_{K_{a}, K_{c}=1,1 \rightarrow 1_{0,1}}$ & 15 & $\ldots$ & $\ldots$ & 5.8637 & 16.55 & $\ldots$ \\
$\mathrm{CH}_{3} \mathrm{OH}$ & $J_{K}=2-1 \rightarrow 1_{-1} E$ & $\ldots$ & 2 & 1 & $\ldots$ & $\ldots$ & 1.2134 \\
& $J_{K}=2_{0} \rightarrow 1_{0} A$ & $\ldots$ & 1 & 2 & $\ldots$ & $\ldots$ & 1.6170 \\
& $J_{K}=2_{0} \rightarrow 1_{0} E$ & $\ldots$ & 2 & 1 & $\ldots$ & $\ldots$ & 1.6166 \\
\hline
\end{tabular}

overabundance of $A$-methanol at low temperatures (Friberg et al. 1988; Wirström et al. 2011). Thus, the integrated intensity of the transition $J_{K}=2_{0} \rightarrow 1_{0} A$ was weighted by 0.69 . Assuming that $f$ (filling factor) equals 1.0, the remaining free parameters in Equation (5) are the beam-averaged column density and the rotational temperature of $\mathrm{CH}_{3} \mathrm{OH}$. By fitting the calculated integrated intensities, from Equation (5), to the observed integrated intensities of the three different methanol transitions, we can find the best solution for the beam-averaged column density and the rotational temperature. The fitting procedure was carried out in IDL using the MPFITFUN package (Markwardt 2009). At the peak position of MM1, the obtained beam-averaged column density and rotational temperature are $1.1 \times 10^{14} \mathrm{~cm}^{-2}$ and $12 \mathrm{~K}$. The optical depth for the transition $J_{K}=2_{0} \rightarrow 1_{0} A$ is 0.1 .

To estimate the molecular abundances with respect to $\mathrm{H}_{2}$, we take the ratio between the column density of a given molecule and the $\mathrm{H}_{2}$ column density derived from the $1.2 \mathrm{~mm}$ dust continuum emission. We use the $1.2 \mathrm{~mm}$ continuum for deriving the $\mathrm{H}_{2}$ column density, rather than the $3.3 \mathrm{~mm}$ continuum, because the emission is more extended, more closely matches the spatial extent of the molecular line emission, and is detected in the regions of interest (for example, where $\mathrm{SiO}$ is detected). Column densities and abundances for all molecules were calculated for the peak positions of $\mathrm{NH}_{2} \mathrm{D}$ (center of $\mathrm{MM} 1$ clump). Additional $\mathrm{SiO}$ column densities and abundances were calculated for the positions marked in Figure 5 ("north," "center-west," and "south").

Uncertainties from the line fitting and dust temperature will propagate to the derived column densities and abundances. At the end, uncertainties in the molecular abundances range between $\sim 35 \%$ and $\sim 50 \%$. All calculated column densities and abundances with their respective uncertainties are summarized in Table 3.

\subsection{The Puzzling $\mathrm{SiO}$ and $\mathrm{CH}_{3} \mathrm{OH}$ Emission}

\subsection{1. $\mathrm{SiO}$}

$\mathrm{SiO}$ is heavily depleted onto dust grains in quiescent regions while it is enhanced in active regions with shocked gas (MartinPintado et al. 1992; Schilke et al. 1997; Caselli et al. 1997). The sputtering and/or grain destruction by shock waves inject $\mathrm{Si}$ atoms that are rapidly oxidized into $\mathrm{SiO}$ and/or Si-bearing species that subsequently form $\mathrm{SiO}$ molecules in the gas phase. In the quiescent gas of dark clouds (in low-mass star-forming regions) the $\mathrm{SiO}$ abundance has an upper limit of $10^{-12}$ (e.g., Jiménez-Serra et al. 2005; Requena-Torres et al. 2007), as expected if the ambient gas has not been significantly affected by ejection of material from dust grains. On the other hand, in regions with active star formation that have molecular outflows, the $\mathrm{SiO}$ abundance in the high-velocity gas can be up to $10^{6}$ times higher than in the quiescent gas (e.g., in L1448; MartinPintado et al. 1992). The large variations of $\mathrm{SiO}$ abundances make this molecule a powerful tracer of shocks. In IRDCs, SiO abundances are in the range $(0.1-1) \times 10^{-9}$ (Sanhueza et al. 2012; Vasyunina et al. 2011). Because IRDC G028.23-00.19 appears to lack embedded IR sources that can drive molecular outflows and produce shocks, the detection of $\mathrm{SiO}$ is highly unexpected.

The $\mathrm{SiO}$ emission in IRDC G028.23-00.19 comprises two different components with different kinematics and spatial distribution. This suggests that the shocks responsible for releasing $\mathrm{SiO}$ and/or $\mathrm{Si}$-bearing species to the gas phase may have two different origins. The $\mathrm{SiO}$ component with broad line widths, located in the southern and northern regions of the IRDC, could be produced by molecular outflows of intermediate-mass stars or clusters of low-mass stars, while the $\mathrm{SiO}$ component with narrow line widths, located in the centerwest part of the IRDC, could be produced by the collision of the subclouds or by the outflows of a few low-mass stars. In the following paragraphs, we develop the idea of $\mathrm{SiO}$ having different origins in different regions of the IRDC.

In the southern and northern regions, line widths are $\gtrsim 6 \mathrm{~km} \mathrm{~s}^{-1}$, which are typical values found toward molecular outflows in star-forming regions. Both $\mathrm{SiO}$ components with broad line widths show no evident wing emission that could allow us to identify the blueshifted and redshifted lobes. The molecular outflows, if present, could be oriented along the line of sight, or the presence of multiple unresolved outflows can also make the identification of wings difficult. In the southern region, the $\mathrm{SiO}$ abundance in the peak position is $3.8 \times 10^{-9}$. This value is typical for regions with molecular outflows (e.g., Jiménez-Serra et al. 2010; Su et al. 2007). In the northern region, the $\mathrm{SiO}$ abundance is $7.5 \times 10^{-10}$. This value is at the low end of abundances found in molecular outflows toward IRDCs (e.g., Jiménez-Serra et al. 2010). Thus, there is evidence supporting the idea that the $\mathrm{SiO}$ component with broad line widths is produced by molecular outflows. However, although the $\mathrm{SiO}$ emission in the northern region is associated with a weak $1.2 \mathrm{~mm}$ peak, the $\mathrm{SiO}$ emission in the southern region is not associated with any core/clump at millimeter emission. As determined by fitting the SED using Herschel observations, the bolometric luminosities encompassing the northern and southern $\mathrm{SiO}$ region are $\sim 300-500 L_{\odot}$ (A. Guzmán 2013, private communication). Due to the low bolometric luminosities, we rule out that the $\mathrm{SiO}$ emission in these regions is being produced by molecular outflows from deeply embedded high-mass stars. Based on the values of the line widths, abundances, and luminosities, we suggest that the shocks releasing $\mathrm{SiO}$ into the gas phase are produced by molecular outflows of deeply embedded intermediate-mass stars or clusters of low-mass stars.

In the center-west region of the IRDC, $\mathrm{SiO}$ line widths are significantly lower $\left(\sim 2 \mathrm{~km} \mathrm{~s}^{-1}\right)$ and comparable to other quiescent gas tracers like $\mathrm{HN}^{13} \mathrm{C}, \mathrm{C}_{2} \mathrm{H}$, and $\mathrm{H}^{13} \mathrm{CO}^{+}$. This $\mathrm{SiO}$ component with narrow line widths shows no detectable line wing emission. Not only that, the $\mathrm{SiO}$ abundance $\left(4.3 \times 10^{-11}\right)$ is low and only a factor of 10 more abundant than the upper 
limits measured in the quiescent gas of molecular dark clouds (Requena-Torres et al. 2007). These characteristics suggest that molecular outflows from intermediate to high-mass stars may not be causing the shocks releasing $\mathrm{SiO}$ to the gas phase. The shocks that release this small amount of $\mathrm{SiO}$ into the gas phase toward the center-west region are likely generated by lowvelocity shocks (Jiménez-Serra et al. 2008, 2010). As discussed below, these shocks may be caused by either the interaction of the subclouds or a few low-mass stars.

The SiO component with narrow line widths is remarkably coincident with the subcloud-subcloud interface (see right panel in Figure 5). As reported by Jiménez-Serra et al. (2010), velocities slightly larger than $10 \mathrm{~km} \mathrm{~s}^{-1}$ are required to sputter $\mathrm{SiO}$ from the mantles of dust grains in low-velocity C-shocks. However, this velocity threshold is above the measured threedimensional relative velocity between subclouds $\left(\sim 2.4 \mathrm{~km} \mathrm{~s}^{-1}\right.$ assuming one-dimensional velocity of $1.4 \mathrm{~km} \mathrm{~s}^{-1}$ in each direction). An alternative mechanism for the production of narrow $\mathrm{SiO}$ in IRDC G028.23-00.19 would be icy mantle vaporization by grain-grain collisions similar to what has been proposed for the IRDC G035.39-00.33 (Caselli et al. 1997; Henshaw et al. 2013). As noted by these authors, ice mantle mixtures of $\mathrm{H}_{2} \mathrm{O}$ :CO have significantly smaller binding energies than pure water ices (by a factor of five; Öberg et al. 2005), and therefore require smaller vaporization threshold velocities (i.e., $2.2 \mathrm{~km} \mathrm{~s}^{-1}$, compared to $6.5 \mathrm{~km} \mathrm{~s}^{-1}$ for pure water ice; see Tielens et al. 1994). The narrow $\mathrm{SiO}$ emission detected toward the overlapping region between subclouds could thus be produced by the vaporization of icy mantles in grain-grain collisions. Therefore, the narrow $\mathrm{SiO}$ emission would not be associated with star formation activity; instead, it would be a signature of a subcloud-subcloud collision. We note, however, that the narrow $\mathrm{SiO}$ component arises only from gas at the velocity corresponding to that of the redshifted subcloud, although this could be a sensitivity effect.

Alternatively, the $\mathrm{SiO}$ component with narrow line widths could be also produced by an unresolved population of a few low-mass stars. In this scenario, due to the distance of the IRDC, beam dilution would prevent us from detecting $\mathrm{SiO}$ wing emission produced by molecular outflows of the low-mass stars. To inspect this scenario, we scaled the $\mathrm{SiO}$ emission of a low-mass star-forming region located in NGC 1333 (350 pc) to the distance of the IRDC G028.23-00.19 (5.1 kpc). Using $1.2 \mathrm{~mm}$ continuum emission, Lefloch et al. (1998a) observed the complex SVS13 and IRAS4A-B, located in NGC 1333, determining a total mass of $12.6 M_{\odot}$ for the three low-mass cores. The angular size of the complex SVS13 and IRAS4A-B at the distance of the IRDC is about the same size of the CARMA synthesized beam $\left(\sim 11^{\prime \prime}\right)$. Lefloch et al. (1998b) observed the same region in $\mathrm{SiO} J=2 \rightarrow 1$. Scaling the $\mathrm{SiO}$ emission to the distance of the IRDC, we find that 1.4 times the emission from a low-mass star-forming region like the SVS13 and IRAS4A-B complex could account for the $\mathrm{SiO}$ emission with narrow line widths obtained in one CARMA synthesized beam. Thus, the origin of the $\mathrm{SiO}$ emission with narrow line widths could be also explained by an unresolved population of a few low-mass stars.

Although higher angular resolution interferometric observations are needed to clearly establish the origin of the $\mathrm{SiO}$ emission, the component with broad $\mathrm{SiO}$ line widths and high $\mathrm{SiO}$ abundances is consistent with molecular outflows from asof-yet-undetected intermediate-mass stars or clusters of lowmass stars. On the other hand, the component with narrow $\mathrm{SiO}$ line widths and low $\mathrm{SiO}$ abundances is consistent with both subcloud-subcloud collision and an unresolved population of a few low-mass stars.

$$
\text { 4.3.2. } \mathrm{CH}_{3} \mathrm{OH}
$$

Currently, the favored formation path of $\mathrm{CH}_{3} \mathrm{OH}$ is primarily through successive hydrogenation of $\mathrm{CO}$ on grain mantles (Watanabe \& Kouchi 2002; Fuchs et al. 2009). In dark clouds, purely gas-phase reactions produce negligible $\mathrm{CH}_{3} \mathrm{OH}$ abundances $\left(\lesssim 10^{-14}\right.$; Garrod et al. 2006). Methanol is predominantly formed on grain surfaces and, then in more evolved regions, it is released into the gas phase mostly by heating from protostars or sputtering of the grain mantles produced by molecular outflows. The study of van der Tak et al. (2000) found three types of $\mathrm{CH}_{3} \mathrm{OH}$ abundances in massive star-forming regions: $\sim 10^{-9}$ for the coldest sources, between $10^{-9}$ and $10^{-7}$ for warmer sources, and $\sim 10^{-7}$ for hot cores. Methanol has also been observed in IRDCs that show signs of star formation (e.g., Sakai et al. 2010, 2008; Gómez et al. 2011). Because IRDC G028.23-00.19 has no detection of embedded IR sources that can heat the environment or drive molecular outflows, the detection of $\mathrm{CH}_{3} \mathrm{OH}$ is highly unexpected. This is the first detection of extended methanol emission in a massive starless clump, although it has been previously detected in low-mass starless cores (e.g., in TMC-1 and L134N; Friberg et al. 1988; Dickens et al. 2000).

As in the case of $\mathrm{SiO}, \mathrm{CH}_{3} \mathrm{OH}$ emission also presents two different components with different kinematics and spatial distributions. This suggests that there may be two different mechanisms releasing methanol to the gas phase. The $\mathrm{CH}_{3} \mathrm{OH}$ emission with broad line widths, co-located with the $\mathrm{SiO}$ emission with broad line widths, could be produced by molecular outflows. The $\mathrm{CH}_{3} \mathrm{OH}$ emission with narrow line widths, located in the center region of the IRDC (including MM1 and the $\mathrm{SiO}$ region with narrow line widths), could be produced by a non-thermal mechanism (e.g., cosmic ray, UV-photons), other than shocks. We discard the notion that thermal evaporation of methanol can occur anywhere in IRDC G028.23-00.19 based on the nondetection of embedded IR sources that could heat the environment. $\mathrm{CH}_{3} \mathrm{OH}$ evaporates at higher temperatures $(\sim 80-100 \mathrm{~K}$; Brown \& Bolina 2007; Green et al. 2009) than those measured in this IRDC. Although a protostar could exist deeply embedded in the cloud and be undetected in the current IR observations, the methanol emission is widespread and the effective heating area of such a protostar would be too small to account for the spatial extension of the $\mathrm{CH}_{3} \mathrm{OH}$ emission.

In the southern and northern regions of the IRDC, where both $\mathrm{CH}_{3} \mathrm{OH}$ and $\mathrm{SiO}$ have broad lines, $\mathrm{CH}_{3} \mathrm{OH}$ line widths are $\gtrsim 4 \mathrm{~km} \mathrm{~s}^{-1}$. Because the methanol transitions are blended, we cannot fit the lines and use the rotational diagram technique to determine column densities. We conclude that in these regions $\mathrm{CH}_{3} \mathrm{OH}$ column densities and abundances are higher than in MM1 (i.e., greater than $1.1 \times 10^{14} \mathrm{~cm}^{-2}$ and $2.7 \times 10^{-9}$ ) based on the comparison of the integrated intensities and apparently larger optical depths. Large line widths and abundances may indicate that in the southern and northern regions of the IRDC, $\mathrm{CH}_{3} \mathrm{OH}$ has been released to the gas phase by outflow activity as, for example, is occurring in IRDC G11.11-0.12 (Gómez et al. 2011).

On the other hand, the methanol emission that emanates from the center of the IRDC cannot be explained by molecular outflows. The line widths are $\sim 2 \mathrm{~km} \mathrm{~s}^{-1}$, typical of regions without outflow activity. The methanol abundance of $2.7 \times 10^{-9}$ is about 1-2 orders of magnitude lower than that observed 
in massive star-forming regions with molecular outflows and one order of magnitude lower than that produced by lowvelocity shocks (Jiménez-Serra et al. 2005). From all the non-thermal mechanisms (e.g., cosmic ray, UV photons) that could explain the observed methanol abundances in the central region of IRDC G028.23-00.19, the exothermicity of surface addition reactions is likely the process that better reproduces the observed abundances. In this mechanism, introduced by Garrod et al. (2006) and further investigated by Garrod et al. (2007), the chemical energy released from the grain-surface addition reactions is able to break the methanol-surface bound, yielding methanol abundances of $(3-4) \times 10^{-9}$, similar to those measured toward the massive, quiescent clump MM1. The high extinction found toward IRDC G028.23-00.19, and the lack of internal heating sources, makes the non-thermal UV photodesorption of $\mathrm{CH}_{3} \mathrm{OH}$ unlikely. Cosmic-ray-induced desorption, a physical mechanism frequently used in dark cloud models (e.g., Hasegawa \& Herbst 1993), cannot reproduce the measured methanol abundances in IRDC G028.23-00.19 either, because the new cosmic-ray-induced desorption rate for methanol is negligible (Collings et al. 2004; Garrod et al. 2007). Therefore, our observations of IRDC G028.23-00.19 show for the first time that the exothermicity of surface addition reactions can also explain the observed methanol abundances of a massive, IR-quiescent clump (see this process included in a chemical network applied to IRDCs in the recent paper of Vasyunina et al. 2013).

\section{4. $\mathrm{NH}_{2} \mathrm{D}$ and $\mathrm{C}_{2} \mathrm{H}$ : Cold Gas Tracers}

\subsection{1. $\mathrm{NH}_{2} \mathrm{D}$}

Deuterated molecules are highly enhanced in the cold gas of prestellar cores, with respect to the local interstellar $\mathrm{D} / \mathrm{H}$ value of $2.3 \times 10^{-5}$ (Linsky et al. 2006). Enhancements of the $\mathrm{NH}_{2} \mathrm{D} / \mathrm{NH}_{3}$ abundance ratio as high as 0.7 (Pillai et al. 2007) and 0.8 (Busquet et al. 2010) have been measured in prestellar massive cores. In cold gas, the deuterium enrichment is primarily initiated by the reaction $\mathrm{H}_{3}^{+}+\mathrm{HD} \Longleftrightarrow \mathrm{H}_{2} \mathrm{D}^{+}+\mathrm{H}_{2}+\Delta E$, which is exothermic by $\Delta E / k=230 \mathrm{~K}$ (Millar et al. 1989). At the typical temperatures of prestellar cores $(\lesssim 20 \mathrm{~K})$, the reverse reaction becomes negligible and the $\mathrm{H}_{2} \mathrm{D}^{+} / \mathrm{H}_{3}^{+}$abundance ratio (and other molecular $\mathrm{D} / \mathrm{H}$ ratios) is larger than the interstellar $\mathrm{D} / \mathrm{H}$. The degree of deuteration is even higher when $\mathrm{CO}$ (the main destroyer of $\mathrm{H}_{3}^{+}$and $\mathrm{H}_{2} \mathrm{D}^{+}$) is removed from the gas phase (e.g., Chen et al. 2011; Crapsi et al. 2005).

Among all the observed molecules in this work, $\mathrm{NH}_{2} \mathrm{D}$ has the narrowest profile with a line width of $1.3 \mathrm{~km} \mathrm{~s}^{-1}$ at the peak position. The $\mathrm{NH}_{2} \mathrm{D}$ abundance of $1.3 \times 10^{-9}$ is high and comparable to the usually more abundant $\mathrm{HCO}^{+}$and $\mathrm{HNC}$, and suggests that the deuterated fraction is high. However, without $\mathrm{NH}_{3}$ observations we cannot determine the exact value of the deuterated fraction. Because $\mathrm{NH}_{2} \mathrm{D}$ emission is confined to the central part of the clump MM1 (see Figure 3), this region is probably the coldest and densest part of the IRDC.

$$
\text { 4.4.2. } \mathrm{C}_{2} \mathrm{H}
$$

$\mathrm{C}_{2} \mathrm{H}$ has been known to be a photodissociation region (PDR) tracer (e.g., Fuente et al. 1993). However, Beuther et al. (2008) suggest that this molecule could also be used to study the cold gas associated with the initial conditions of high-mass star formation. Sanhueza et al. (2012) detect $\mathrm{C}_{2} \mathrm{H}$ in $\sim 30 \%$ of their sub-sample composed by IR quiescent clumps. They suggest that mapping the $\mathrm{C}_{2} \mathrm{H}$ distribution at high angular resolution may help to clarify if the $\mathrm{C}_{2} \mathrm{H}$ emission comes from the external photodissociated layers of clumps or if the $\mathrm{C}_{2} \mathrm{H}$ emission comes from the dense, cold gas inside IR quiescent clumps.

As can be seen in Figure 3, the spatial distribution of the $\mathrm{C}_{2} \mathrm{H}$ emission resembles that of $\mathrm{NH}_{2} \mathrm{D}$. In IRDC G028.23-00.19, the $\mathrm{C}_{2} \mathrm{H}$ emission comes from the central and coldest part of the cloud, and not from the external layers of the IRDC. $\mathrm{C}_{2} \mathrm{H}$ and $\mathrm{NH}_{2} \mathrm{D}$ molecules have their peak emission in the same place and their spatial extensions are similar (although $\mathrm{C}_{2} \mathrm{H}$ extends slightly farther out of the clump MM1). Because $\mathrm{C}_{2} \mathrm{H}$ emission is mostly located at the same position as the $\mathrm{NH}_{2} \mathrm{D}$ emission, i.e., the center of MM1, we suggest $\mathrm{C}_{2} \mathrm{H}$ is also tracing dense, cold gas in IRDC G028.23-00.19.

\section{5. $\mathrm{HCO}^{+}, \mathrm{H}^{13} \mathrm{CO}^{+}, \mathrm{HNC}, \mathrm{HN}^{13} \mathrm{C}$, and $\mathrm{N}_{2} \mathrm{H}^{+}$}

$\mathrm{HCO}^{+}$and $\mathrm{HNC}$ abundances are estimated using their isotopologues $\left(\mathrm{H}^{13} \mathrm{CO}^{+}\right.$and $\left.\mathrm{HN}^{13} \mathrm{C}\right)$. The $\mathrm{HCO}^{+}$abundance of $1.2 \times 10^{-9}$ and HNC abundance of $1.5 \times 10^{-9}$ are consistent, within the uncertainties, with the values determined by Sanhueza et al. (2012) in IRDC G028.23-00.19 MM1, using $12 \mathrm{~K}$ in their calculation. The abundances determined in the MM1 clump are about one order of magnitude lower than the typical values found by Sanhueza et al. (2012) in their sample of $\sim 30$ IR, quiescent clumps (median values of $2.4 \times 10^{-8}$ for $\mathrm{HCO}^{+}$and $3.5 \times 10^{-8}$ for $\mathrm{HNC}$ ). They found that $\mathrm{HCO}^{+}$and HNC abundances increase as the clumps evolve, from IR, quiescent clumps to protostellar objects and on to $\mathrm{H}$ iI regions. The low abundances measured in our work for the clump MM1 in IRDC G028.23-00.19 suggest that this clump is in a very early stage of evolution.

The relative intensities of the three hyperfine transitions of $\mathrm{N}_{2} \mathrm{H}^{+} J=1 \rightarrow 0$ are 1:3:5, with the brightest line in the center $\left(J F_{1} F=123 \rightarrow 012\right)$. However, toward the peak position of IRDC G028.23-00.19 (see Figure 6) and on a large region of the IRDC, the brightest hyperfine line is the one with the lowest relative intensity ratio $\left(J F_{1} F=101 \rightarrow 012\right)$. This peculiar intensity ratio suggests a very high optical depth for $\mathrm{N}_{2} \mathrm{H}^{+}$. Thus, the $\mathrm{N}_{2} \mathrm{H}^{+}$abundance of $4.3 \times 10^{-10}$ is a lower limit.

\subsection{The Role of the Subclouds}

Because line widths in IRDCs are narrower than those in molecular clouds with copious star formation, IRDCs are excellent laboratories to study large sub-structures inside molecular clouds, such as subclouds or filaments. Jiménez-Serra et al. (2010) and Devine et al. (2011) also find close velocity components of $\lesssim 2 \mathrm{~km} \mathrm{~s}^{-1}$ in two different IRDCs. Jiménez-Serra et al. (2010) find three subclouds in IRDC G035.39-00.33 using IRAM single-dish observations of $\mathrm{C}^{18} \mathrm{O}$. They find a widespread narrow $\mathrm{SiO}$ emission in this IRDC that could have been produced by the interaction of the subclouds. In another IRDC, G019.30+00.07, Devine et al. (2011) find three subclouds using VLA observations of $\mathrm{NH}_{3}$ and CCS. They find that $\mathrm{NH}_{3}$ and CCS trace different parts of the subclouds: $\mathrm{NH}_{3}$ peaks in the densest regions and CCS peaks in the subcloud-subcloud and outflow-subcloud interfaces. They suggest that in these boundary regions the gas is chemically young due to collisions that release molecules from dust grains to the gas phase, leading to the presence of early time molecules like CCS.

We find that two subclouds form IRDC G028.23-00.19. Notably, the narrow $\mathrm{SiO}$ component is located in the region where both subclouds overlap. Assuming that this $\mathrm{SiO}$ emission is produced by the collision of the subclouds, this work adds new evidence in favor of the idea that the subclouds or filaments 
recently found in IRDCs are interacting and are part of the same cloud, and not just a superposition of clouds along the line of sight. Thus, it is not simply a coincidence that IRDCs frequently show no evident signs of active star formation; IRDCs may be in a very early stage in which the clouds are still being assembled.

\subsection{The "Prestellar" Nature of IRDC G028.23-00.19}

Except for the bright unrelated OH/IR star superimposed against the northern part of the cloud (Bowers \& Knapp 1989), IRDC G028.23-00.19 is IR dark from 3.6 to $70 \mu \mathrm{m}$. The absence of embedded IR sources suggests that the whole IRDC is in an early stage of evolution, without signs of active star formation. However, our CARMA observations show broad $\mathrm{SiO}$ and $\mathrm{CH}_{3} \mathrm{OH}$ emission in the northern and southern regions of the IRDC that may indicate molecular outflow activity and, consequently, current star formation. In addition, one of the possible explanations for the narrow $\mathrm{SiO}$ component in the center-west part of the IRDC is that the $\mathrm{SiO}$ emission is produced for an unresolved population of low-mass stars.

On the other hand, the central clump MM1 is quiescent. It is cold $(12 \mathrm{~K})$ and lacks IR sources, centimeter continuum and $\mathrm{SiO}$ emission are not detected, molecular line widths are narrow $\left(\lesssim 2 \mathrm{~km} \mathrm{~s}^{-1}\right)$, and the $\mathrm{NH}_{2} \mathrm{D}$ abundance is high. The combination of all these characteristics of MM1 indicates that this clump is still in a prestellar stage. Although regions in the same IRDC may show indirect signs of star formation, all the observations are consistent with the massive clump MM1 being a pristine starless clump. To determine whether MM1 will form stars in the future, we compare the dust mass $\left(M_{\text {dust }}\right)$ with the virial mass $\left(M_{\text {vir }}\right)$. In Section 4.1 we estimated an $M_{\text {dust }}$ of $1520 M_{\odot}$, using the $1.2 \mathrm{~mm}$ dust continuum emission. To determine the virial mass, we follow the prescription of MacLaren et al. (1988). The virial mass of a clump with a uniform density profile, neglecting magnetic fields and external forces, is given by $M_{\mathrm{vir}}=210 R \Delta V^{2} M_{\odot}$, where $R$ is the radius of the clump in $\mathrm{pc}$ and $\Delta V$ is the line width in $\mathrm{km} \mathrm{s}^{-1}$. Adopting the same size used to obtain $M_{\text {dust }}$, a radius of $0.6 \mathrm{pc}$, and an average line width for MM1 of $1.9 \mathrm{~km} \mathrm{~s}^{-1}$ (from Table 2), we obtain a virial mass of $\sim 450 M_{\odot}$. Therefore, the virial parameter, $\alpha=M_{\text {vir }} / M_{\text {dust }}$, is 0.3 , indicating that the clump MM1 is gravitationally bound and eventually, due to its large mass, will form stars. To further investigate if the clump MM1 will form high-mass stars, we use the results from Kauffmann \& Pillai (2010). They find an empirical massive star formation threshold, based on clouds with and without high-mass star formation. They suggest that IRDCs with masses larger than the mass limit given by $m_{\mathrm{lim}}=$ $870 M_{\odot}(r / p c)^{1.33}$ are forming high-mass stars or will form in the future. Applying this relationship to MM1, its corresponding mass limit is $440 M_{\odot}$. Thus, "the compactness" $\left(M_{\text {dust }} / m_{\text {lim }}\right)$ of MM1 is 3.5 and it is highly likely that the clump will form high-mass stars in the future.

Our observation that other regions in the IRDC, but not MM1, may be forming stars raises a number of questions. Why would star formation not start first in MM1? If we observe at higher angular resolution and sensitivity, would we be able to resolve and associate cores or protostars with the $\mathrm{SiO}$ emission? How does the scenario observed in IRDC G028.23-00.19 fit into current models of high-mass star formation? Such questions may be possibly answered with observations at higher angular resolution and better sensitivity.

\section{CONCLUSIONS}

We have observed the IRDC G028.23-00.19 in several molecular species $\left(\mathrm{NH}_{2} \mathrm{D}, \mathrm{H}^{13} \mathrm{CO}^{+}, \mathrm{SiO}, \mathrm{HN}^{13} \mathrm{C}, \mathrm{C}_{2} \mathrm{H}\right.$, $\mathrm{HCO}^{+}, \mathrm{HNC}, \mathrm{N}_{2} \mathrm{H}^{+}$, and $\mathrm{CH}_{3} \mathrm{OH}$ ) and continuum emission at $3.3 \mathrm{~mm}$ using CARMA (11" angular resolution). This IRDC is dark at Spitzer 3.6, 4.5, 8.0, and $24 \mu \mathrm{m}$ and Herschel $70 \mu \mathrm{m}$. In its center, the IRDC hosts one of the most massive IR, quiescent clumps known (MM1). We have examined the spectral line observations and draw the following conclusions.

1. Using the emission detected at 250, 350, and $500 \mu \mathrm{m}$ with Herschel, and the $1.2 \mathrm{~mm}$ emission from IRAM $30 \mathrm{~m}$, we updated the mass $\left(1520 M_{\odot}\right)$ and the dust temperature (12 K) of the central clump in IRDC G028.23-00.19.

2. The low temperature, high $\mathrm{NH}_{2} \mathrm{D}$ abundance, low $\mathrm{HCO}^{+}$ and $\mathrm{HNC}$ abundances, non-detection of $\mathrm{SiO}$, narrow line widths, and absence of embedded IR sources in MM1 indicate that the clump still remains in a prestellar phase.

3. Strong $\mathrm{SiO}$ components with broad line widths are detected in the northern and southern regions of the IRDC. The large line widths $\left(6-9 \mathrm{~km} \mathrm{~s}^{-1}\right)$ and high abundances $\left((0.8-3.8) \times 10^{-9}\right)$ of $\mathrm{SiO}$ suggest that the mechanism releasing $\mathrm{SiO}$ from the dust grains into the gas phase could be molecular outflows from undetected intermediate-mass stars or clusters of low-mass stars deeply embedded in the IRDC. However, in the southern region where the $\mathrm{SiO}$ abundance peaks, there is no associated counterpart in the continuum.

4. A weaker $\mathrm{SiO}$ component is detected in the center-west part of the IRDC. The narrow line widths $\left(\sim 2 \mathrm{~km} \mathrm{~s}^{-1}\right)$ and low $\mathrm{SiO}$ abundances $\left(4.3 \times 10^{-11}\right)$ are consistent with either a "subcloud-subcloud" interaction or an unresolved population of a few low-mass stars. Higher angular resolution observations are needed to clearly establish the origin of the narrow $\mathrm{SiO}$ emission.

5. We report unexpected widespread $\mathrm{CH}_{3} \mathrm{OH}$ emission throughout the whole IRDC and the first detection of extended methanol emission in a massive prestellar clump. Because IR observations show no primary protostar embedded in the IRDC, we reject thermal evaporation of methanol from dust mantles as the production mechanism. $\mathrm{CH}_{3} \mathrm{OH}$ emission with broad line widths supports the idea of molecular outflows in the southern and northern regions of the IRDC. The $\mathrm{CH}_{3} \mathrm{OH}$ emission at the position of MM1 is rather narrow. We suggest that the most likely mechanism able to release methanol from the dust grains to the gas phase in such a cold region is the exothermicity of surface reactions (Garrod et al. 2006, 2007).

6. $\mathrm{C}_{2} \mathrm{H}$ has been suggested to be a PDR tracer. However, recent evidence suggests it could also trace cold, dense gas associated with earlier stages of star formation. Because the spatial distribution of $\mathrm{C}_{2} \mathrm{H}$ emission resembles that of $\mathrm{NH}_{2} \mathrm{D}$, we suggest $\mathrm{C}_{2} \mathrm{H}$ traces cold, dense gas in this IRDC.

7. $\mathrm{HN}^{13} \mathrm{C}$ reveals that the IRDC is composed of two substructures ("subclouds") separated by $1.4 \mathrm{~km} \mathrm{~s}^{-1}$. Remarkably, the subclouds overlap in the center-west region of the IRDC, exactly coincident with the narrow $\mathrm{SiO}$ component. One explanation for the narrow $\mathrm{SiO}$ component is that both subclouds may be interacting and producing low-velocity shocks that release small amounts of $\mathrm{SiO}$ to the gas phase. We speculate that IRDCs may be young molecular clouds that could still be in a stage where they are being assembled. 
8. In the densest part of the IRDC, the MM1 clump shows no signs of star formation. Notably, in other regions of the IRDC it appears that star formation activity may be occurring. Why star formation would begin in less dense regions and how the findings on IRDC G028.23-00.19 fit into current models of high-mass star formation are open questions that will be addressed in future investigations.

P.S. gratefully acknowledges the instructors of the CARMA Summer School 2011, especially for their enthusiasm and dedication to the professors John Carpenter, Nikolaus Volgenau, Melvyn Wright, Dick Plambeck, and Marc Pound. P.S. thanks Andrés Guzmán for his careful reading of the paper. We also thank the anonymous referee for helpful comments that improved the paper. P.S. and J.M.J. acknowledge funding support from NSF grant No. AST-0808001 and AST-1211844. I.J.-S. acknowledges the financial support from the People Programme (Marie Curie Actions) of the European Union's Seventh Framework Programme (FP7/2007-2013) under REA grant agreement number PIIF-GA-2011-301538. Support for CARMA construction was derived from the states of California, Illinois, and Maryland, the James S. McDonnell Foundation, the Gordon and Betty Moore Foundation, the Kenneth T. and Eileen L. Norris Foundation, the University of Chicago, the Associates of the California Institute of Technology, and the National Science Foundation. Ongoing CARMA development and operations are supported by the National Science Foundation under a cooperative agreement, and by the CARMA partner universities.

\section{REFERENCES}

André, P., Basu, S., \& Inutsuka, S. 2009, in Structure Formation in Astrophysics, ed. G. Chabrier (Cambridge: Cambridge Univ. Press), 254

Battersby, C., Bally, J., Jackson, J. M., et al. 2010, ApJ, 721, 222

Benjamin, R. A., Churchwell, E., Babler, B. L., et al. 2003, PASP, 115, 953

Beuther, H., Semenov, D., Henning, T., \& Linz, H. 2008, ApJL, 675, L33

Bowers, P. F., \& Knapp, G. R. 1989, ApJ, 347, 325

Brown, W. A., \& Bolina, A. S. 2007, MNRAS, 374, 1006

Busquet, G., Palau, A., Estalella, R., et al. 2010, A\&A, 517, L6

Carey, S. J., Clark, F. O., Egan, M. P., et al. 1998, ApJ, 508, 721

Carey, S. J., Feldman, P. A., Redman, R. O., et al. 2000, ApJL, 543, L157

Carey, S. J., Noriega-Crespo, A., Mizuno, D. R., et al. 2009, PASP, 121, 76

Caselli, P., Hartquist, T. W., \& Havnes, O. 1997, A\&A, 322, 296

Chambers, E. T., Jackson, J. M., Rathborne, J. M., \& Simon, R. 2009, ApJS, 181,360

Chen, H.-R., Liu, S.-Y., Su, Y.-N., \& Wang, M.-Y. 2011, ApJ, 743, 196

Chira, R.-A., Beuther, H., Linz, H., et al. 2013, A\&A, 552, A40

Collings, M. P., Anderson, M. A., Chen, R., et al. 2004, MNRAS, 354, 1133

Crapsi, A., Caselli, P., Walmsley, C. M., et al. 2005, ApJ, 619, 379

Daniel, F., Cernicharo, J., \& Dubernet, M.-L. 2006, ApJ, 648, 461

Devine, K. E., Chandler, C. J., Brogan, C., et al. 2011, ApJ, 733, 44

Dickens, J. E., Irvine, W. M., Snell, R. L., et al. 2000, ApJ, 542, 870

Egan, M. P., Shipman, R. F., Price, S. D., et al. 1998, ApJL, 494, L199

Fontani, F., Palau, A., Caselli, P., et al. 2011, A\&A, 529, L7

Friberg, P., Hjalmarson, A., Madden, S. C., \& Irvine, W. M. 1988, A\&A, 195,281

Fuchs, G. W., Cuppen, H. M., Ioppolo, S., et al. 2009, A\&A, 505, 629

Fuente, A., Martin-Pintado, J., Cernicharo, J., \& Bachiller, R. 1993, A\&A, 276,473

Garay, G., \& Lizano, S. 1999, PASP, 111, 1049

Garden, R. P., Hayashi, M., Hasegawa, T., Gatley, I., \& Kaifu, N. 1991, ApJ, 374,540

Garrod, R., Park, I. H., Caselli, P., \& Herbst, E. 2006, FaDi, 133, 51

Garrod, R. T., Wakelam, V., \& Herbst, E. 2007, A\&A, 467, 1103
Girart, J. M., Viti, S., Williams, D. A., Estalella, R., \& Ho, P. T. P. 2002, A\&A, 388,1004

Gómez, L., Wyrowski, F., Pillai, T., Leurini, S., \& Menten, K. M. 2011, A\&A, 529, A161

Green, S. D., Bolina, A. S., Chen, R., et al. 2009, MNRAS, 398, 357

Hasegawa, T. I., \& Herbst, E. 1993, MNRAS, 261, 83

Henshaw, J. D., Caselli, P., Fontani, F., et al. 2013, MNRAS, 428, 3425

Hoq, S., Jackson, J. M., Foster, J. B., et al. 2013, ApJ, submitted

Jackson, J. M., \& Kraemer, K. E. 1999, ApJ, 512, 260

Jiménez-Serra, I., Caselli, P., Martín-Pintado, J., \& Hartquist, T. W. 2008, A\&A, 482,549

Jiménez-Serra, I., Caselli, P., Tan, J. C., et al. 2010, MNRAS, 406, 187

Jiménez-Serra, I., Martín-Pintado, J., Rodríguez-Franco, A., \& Martín, S. 2005, ApJL, 627, L121

Kauffmann, J., \& Pillai, T. 2010, ApJL, 723, L7

Kim, G., Lee, C. W., Kim, J., et al. 2010, JKAS, 43, 9

Lefloch, B., Castets, A., Cernicharo, J., Langer, W. D., \& Zylka, R. 1998a, A\&A, 334, 269

Lefloch, B., Castets, A., Cernicharo, J., \& Loinard, L. 1998b, ApJL, 504, L109

Linsky, J. L., Draine, B. T., Moos, H. W., et al. 2006, ApJ, 647, 1106

MacLaren, I., Richardson, K. M., \& Wolfendale, A. W. 1988, ApJ, 333, 821

Markwardt, C. B. 2009, in ASP Conf. Ser. 411, Astronomical Data Analysis Software and Systems XVIII, ed. D. A. Bohlender, D. Durand, \& P. Dowler (San Francisco, CA: ASP), 251

Martin-Pintado, J., Bachiller, R., \& Fuente, A. 1992, A\&A, 254, 315

Miettinen, O., Hennemann, M., \& Linz, H. 2011, A\&A, 534, A134

Milam, S. N., Savage, C., Brewster, M. A., Ziurys, L. M., \& Wyckoff, S. 2005, ApJ, 634, 1126

Millar, T. J., Bennett, A., \& Herbst, E. 1989, ApJ, 340, 906

Molinari, S., Swinyard, B., Bally, J., et al. 2010, A\&A, 518, L100

Müller, H. S. P., Schlöder, F., Stutzki, J., \& Winnewisser, G. 2005, JMoSt, 742,215

Müller, H. S. P., Thorwirth, S., Roth, D. A., \& Winnewisser, G. 2001, A\&A, 370, L49

Öberg, K. I., van Broekhuizen, F., Fraser, H. J., et al. 2005, ApJL, 621, L33

Ossenkopf, V., \& Henning, T. 1994, A\&A, 291, 943

Perault, M., Omont, A., Simon, G., et al. 1996, A\&A, 315, L165

Peretto, N., \& Fuller, G. A. 2009, A\&A, 505, 405

Pickett, H. M., Poynter, R. L., Cohen, E. A., et al. 1998, JQSRT, 60, 883

Pillai, T., Kauffmann, J., Wyrowski, F., et al. 2011, A\&A, 530, A118

Pillai, T., Wyrowski, F., Carey, S. J., \& Menten, K. M. 2006, A\&A, 450, 569

Pillai, T., Wyrowski, F., Hatchell, J., Gibb, A. G., \& Thompson, M. A. 2007, A\&A, 467, 207

Pineda, J. E., \& Teixeira, P. S. 2013, A\&A, 555, A106

Ragan, S. E., Bergin, E. A., \& Wilner, D. 2011, ApJ, 736, 163

Rathborne, J. M., Jackson, J. M., Chambers, E. T., et al. 2010, ApJ, 715, 310

Rathborne, J. M., Jackson, J. M., \& Simon, R. 2006, ApJ, 641, 389

Rathborne, J. M., Jackson, J. M., Zhang, Q., \& Simon, R. 2008, ApJ, 689, 1141

Requena-Torres, M. A., Marcelino, N., Jiménez-Serra, I., et al. 2007, ApJL, $655, \mathrm{~L} 37$

Sakai, T., Sakai, N., Furuya, K., et al. 2012, ApJ, 747, 140

Sakai, T., Sakai, N., Hirota, T., \& Yamamoto, S. 2010, ApJ, 714, 1658

Sakai, T., Sakai, N., Kamegai, K., et al. 2008, ApJ, 678, 1049

Sanhueza, P., Garay, G., Bronfman, L., et al. 2010, ApJ, 715, 18

Sanhueza, P., Jackson, J. M., Foster, J. B., et al. 2012, ApJ, 756, 60

Schilke, P., Walmsley, C. M., Pineau des Forets, G., \& Flower, D. R. 1997, A\&A, 321, 293

Simon, R., Jackson, J. M., Rathborne, J. M., \& Chambers, E. T. 2006, ApJ, 639,227

Su, Y.-N., Liu, S.-Y., Chen, H.-R., Zhang, Q., \& Cesaroni, R. 2007, ApJ, 671,571

Tielens, A. G. G. M., McKee, C. F., Seab, C. G., \& Hollenbach, D. J. 1994, ApJ, 431,321

van der Tak, F. F. S., van Dishoeck, E. F., \& Caselli, P. 2000, A\&A, 361, 327

Vasyunina, T., Linz, H., Henning, T., et al. 2011, A\&A, 527, A88

Vasyunina, T., Vasyunin, A. I., Herbst, E., et al. 2013, ApJ, submitted

Wang, K., Zhang, Q., Wu, Y., \& Zhang, H. 2011, ApJ, 735, 64

Wang, Y., Zhang, Q., Rathborne, J. M., Jackson, J., \& Wu, Y. 2006, ApJL, $651, \mathrm{~L} 125$

Watanabe, N., \& Kouchi, A. 2002, ApJL, 571, L173

Wirström, E. S., Geppert, W. D., Hjalmarson, A., et al. 2011, A\&A, 533, A24

Zhang, Q., Wang, Y., Pillai, T., \& Rathborne, J. 2009, ApJ, 696, 268 